\title{
Satisficing Choice Procedures
}

\author{
Mauro Papi \\ School of Economics and Finance (University of St Andrews), \\ Castlecliffe, The Scores, KY169AL St Andrews, Fife, Scotland, UK \\ E-mail:mp65@st-andrews.ac.uk \\ Phone: +44 (0) 1334462427 \\ Fax: +44(0) 1334462444
}

\begin{abstract}
A challenging issue in modern choice theory is how to enrich the kind of data available to the researcher along with our understanding of the decision-making process. We propose an axiomatic characterization of the 'satisficing' heuristic under various informational structures. In particular we examine the cases in which search behavior is both observable, partially observable, and unobservable. We also investigate behavioral definitions of preference and satisfaction and establish uniqueness results. Finally, we relate our framework to several well-known existing models.

Keywords: Bounded Rationality, Consideration Set, Revealed Preference, Satisficing, Search.
\end{abstract}

JEL classification: D01.

\section{Introduction}

The problem of choice is the basis of microeconomic theory. Standard models of choice theory assume that a choice problem is composed of two elements: a family $\mathcal{P}(X)$ of nonempty subsets of the grand set of alternatives $X$ and a choice function $c: \mathcal{P}(X) \rightarrow X$. The problem of choice entails picking one element from a budget set $A \in \mathcal{P}(X)$ that is known in advance (Richter, 1966; Mas-Colell et al., 1995).

This framework has been criticized as unrealistic. According to Herbert Simon,

in most global models of rational choice, all alternatives are evaluated be-

fore a choice is made. In actual human decision-making, alternatives are 
often examined sequentially. We may, or may not, know the mechanism that determines the order of procedure. When alternatives are examined sequentially, we may regard the first satisfactory alternative that is evaluated as such as the one actually selected. (Simon, 1955, 110)

We propose a model within the revealed preference approach in which the decisionmaker (DM) discovers and analyzes alternatives sequentially. In the real world there are many circumstances in which alternatives are presented to the DM in form of a sequence. For instance, alternatives may be disposed horizontally on some shelf and the DM examines them from left to right. In this case there is a spatial constraint that prevents the DM from perceiving all alternatives before deciding. Alternatively, the DM may receive job offers sequentially in time. There are also circumstances in which constraints are neither spatial nor temporal, but the DM examines alternatives sequentially, because alternatives come to the DM's mind according to some ordering. As an example think of a person who has to decide where to spend her summer vacation. Typically, an individual does not have in mind a complete set of potential destinations. Rather, she discovers and analyzes new options by searching on the web and referring to travel agencies while the decision process is taking place.

We assume that the DM behaves according to the satisficing heuristic (Simon, 1955). That is, she explores alternatives sequentially, stops searching as soon as she identifies the first satisfactory alternative, and selects the best alternative among those discovered. This choice is motivated by the fact that there is experimental evidence consistent with the satisficing heuristic (Caplin et al., 2011; Reutskaja et al., 2011).

We investigate the conditions under which we can infer from choices whether or not an alternative is revealed to be satisfactory (revealed satisfaction) and to be preferred to some other alternative (revealed preference). A distinctive feature of our work is that we explore these issues under various informational structures depending on what variables are observable. Over the last decades, new devices have been developed in experimental economics to enrich the data available to the analyst in order to gain a deeper understanding of the mechanisms behind the decision-making process. How to expand the informational structure along with our understanding of the model is key. 
Our work addresses this issue by considering three different choice domains.

We first assume that, for each choice problem, we can observe not only the budget set and the choice made by the DM, but also search order. In this case we provide behavioral definitions of both satisfaction and preference, show that our procedure is equivalent to two simple properties of choice, and illustrate that our model includes standard maximization as a special case.

Under the second domain, we assume that search order is just partially observable. In this case we provide behavioral definitions of satisfaction and preference and axiomatically characterize our procedure by using slightly modified versions of the two properties mentioned above.

Finally, we assume that search order is unobservable. Under this domain we show that we can recover satisfaction and preference, but only over unsatisfactory alternatives. In addition we demonstrate that in this case our model is a special case of the standard maximization procedure and provide a short discussion of our results.

The model is related to the literature on attention and consideration set (Manzini and Mariotti, 2007; Masatlioglu et al., 2009; Eliaz and Spiegler, 2011; Eliaz et al., 2011). The closest studies to our work are Rubinstein and Salant (2006), Tyson (2008), Masatlioglu and Nakajima (2009), Horan (2010), Caplin and Dean (2011), and Raymond (2011). We discuss them in turn.

Rubinstein and Salant (2006) develop a model called choice from lists in which the DM chooses either the first or the last maximal alternative from any list depending on its positioning in the list. In the same spirit, Horan (2010) investigates several sequential search models including the satisficing one by utilizing the choice from lists framework. In contrast, we propose a model in which the DM does not necessarily examine alternatives one by one. More precisely, we consider both the standard case in which the DM examines all alternatives simultaneously, the polar case in which alternatives are examined one by one, and all the cases in between.

Tyson (2008) investigates a particular aspect of the concept of 'satisficing' by proposing a model in which the DM does not fully perceive her preferences and this bias increases in the complexity of the choice problem. He shows that, when complexity it is aligned with set inclusion (nestedness of preferences), then his choice proce- 
dure is equivalent to Sen (1971)'s $\beta$ property. ${ }^{1}$ In contrast, we focus on another aspect of the satisficing theory by assuming that the DM discovers and analyzes alternatives sequentially.

Masatlioglu and Nakajima (2009) propose a model called choice by iterative search in which the DM starts by analyzing an exogenously given contemplation point, considers only those alternatives that are similar to it and, by means of an iterative search process, stops searching as soon as the contemplation point is the best available alternative in the consideration set. In contrast, we assume that the search process terminates as soon as the first satisfactory alternative is identified.

Caplin and Dean (2011) propose a reservation-based search decision strategy by formalizing the concept of choice process data. That is, the DM picks the best alternative among the ones she has already explored at any given point in time and stops searching as soon as she identifies the first alternative that yields at least the reservation utility, given that searching is costly. Unlike Caplin and Dean (2011), we do not make use of choice process data, but consider only final choices and assume that search order is observable or partially observable. We also investigate the case in which search order is unobservable as Caplin and Dean (2011) do, but the two models still differ because unlike them we assume that the choice correspondence records the choices made by the DM under multiple menu sequences.

Raymond (2011) characterizes a broad set of search models, including classical model of sequential search, by assuming that observable data are given by preferences over lists of outcomes. In contrast, we focus on the satisficing model and assume that observable data are choices from within menu sequences.

In addition, in a section below we formally relate our work to the following models: choice from lists (Rubinstein and Salant, 2006), choice with frames (Salant and Rubinstein, 2008), agenda rationalizability (Apesteguia and Ballester, 2010), choice by iterative search (Masatlioglu and Nakajima, 2009), and choice with initial endowments (Masatlioglu and Ok, 2009).

The paper is organized as follows. Section 2 develops the formal model. Section

\footnotetext{
${ }^{1}$ Property $\beta$ : given two alternatives $x, y \in A \subseteq B$, if $x \in C(A)$ and $y \in C(B)$, then $x \in C(B)$.
} 
3 discusses the first informational case by providing motivations and examples, investigates behavioral definitions of satisfaction and preference, and provides an axiomatic characterization of our model (along with uniqueness results) under the first choice domain. Section 4 and Section 5 do the same job under the second and the third domain, respectively. Section 6 investigates the relationships with other models. Section 7 concludes.

\section{The Model}

Let $X$ be a finite grand set of alternatives and $\mathcal{P}(X)$ the set of all non-empty subsets of $X$. Each element $A$ of $\mathcal{P}(X)$ is a budget set. We call menu sequence the sequence with which the DM examine alternatives and formally define it as follows.

Definition 1. A menu sequence $\mathbf{A}$ of the budget set $A \in \mathcal{P}(X)$ is a sequence $\mathbf{A} \equiv$ $\left\langle A_{1}, \ldots, A_{N_{\mathrm{A}}}\right\rangle$ such that $A_{j} \subset A_{k} \subset A$, for all $k>j$ and $A_{N_{\mathrm{A}}}=A$.

We call each element $A_{j}$ of the menu sequence $\mathbf{A}$ stage of the menu sequence $\mathbf{A}$. We assume that the DM can investigate all the alternatives within a stage without any additional cost. In this study we will consider various choice domains depending on how what variables are observable. We first consider the domain $\mathcal{D}_{1}$, according to which we assume that we can observe not only the choice made by the DM and the budget set, but also the entire menu sequence.

Imagine a consumer that receives a sequence of e-mails containing various deals on some product (e.g. phone and broadband). At each stage of the process, the consumer compares multiple offers and decides whether to either purchase one of the available products or wait for further options to come. Another example is a stockbroker that every day has to decide how to invest considerable sums of money on the behalf of the investors. It is reasonable to expect that during the day she will have to repeatedly make quick decisions, such as whether to either (i) buy $x$ shares, or (ii) sell $y$ bonds, or (iii) buy $x$ shares and sell $y$ bonds, or (iv) do nothing and wait. Given the high time pressure within financial markets, in order to maximize the value of the investors' portfolios the stockbroker is somehow forced to compare multiple alternatives simultaneously. 
Other examples of menu sequences are all circumstances in which objects appear in sequential packages, physical spaces, and web-pages. ${ }^{2}$

Formally, let $\mathcal{D}_{1} \subseteq \bigcup_{A \in \mathcal{P}(X)} \Sigma(A)$, where $\Sigma(A)$ is the set of menu sequences for $A \in \mathcal{P}(X)$. At the end of next section we will specify what data (menu sequences with relative choices) we need to observe in order to obtain our results. We call extended choice function the choice function defined on $\mathcal{D}_{1}$. We assume that $c(\mathbf{A}) \in A$ is nonempty and single-valued.

Let $>$ be a strict linear order on the set $X$ representing DM's preferences. ${ }^{3}$ Let $x^{s} \in X$ be a distinguished alternative such that all alternatives to which $x^{s}$ is $>$-preferred are considered to be unsatisfactory. Let $U C_{>}\left(A ; x^{s}\right) \equiv\left\{x \in A \mid \neg\left(x^{s}>x\right)\right\}$ be the uppercontour set of $x^{s}$ according to $>$, representing the set of satisfactory alternatives available in the set $A$. Let $\max (A ;>) \equiv\{x \in A \mid \nexists y \in A$ s.t. $y>x\}$ be the set of maximal alternatives according to the relation $>$ in the set $A$.

Definition 2. $c$ is a Satisficing Choice Function (SCF) if and only if there exist a strict linear order $>$ on $X$ and a distinguished alternative $x^{s} \in X$ such that, for any $\mathbf{A}=$ $\left\langle A_{j}\right\rangle \in \mathcal{D}_{1}$,

$$
\{c(\mathbf{A})\} \equiv \max \left(\Gamma_{(\mathbf{A})} ;>\right)
$$

where $\Gamma_{(\mathbf{A})} \subseteq A$ is a a consideration set mapping

$$
\Gamma_{(\mathbf{A})} \equiv \begin{cases}A_{j^{s}} & \text { if } U C_{>}\left(A ; x^{s}\right) \neq \emptyset \\ A & \text { otherwise }\end{cases}
$$

and $j^{s} \equiv \min \left\{j \mid A_{j} \cap U C_{>}\left(A ; x^{s}\right) \neq \emptyset\right\}$.

\footnotetext{
${ }^{2}$ The above examples fit within the concept of menu sequence, but do not within the Rubinstein and Salant (2006)'s list one.

${ }^{3} \mathrm{~A}$ binary relation $>$ is irreflexive whenever $(x, x) \notin>$. Given $x, y, z \in X$, a binary relation $>$ is transitive, whenever $(x, y) \in>$ and $(y, z) \in>$ imply that $(x, z) \in>$. Given $x, y \in X$ such that $x \neq y$, a binary relation $>$ is complete whenever either $(x, y) \in>$ or $(y, x) \in>$. Given $x, y \in X$, a binary relation $>$ is asymmetric whenever $(x, y) \in>$ implies that $(y, x) \notin>$. A strict linear order is a transitive, asymmetric, irreflexive, and complete binary relation.
} 
The primitive element of our model is the pair $\left(>, x^{s}\right)$, which fully characterizes the DM. Our interpretation is that the DM has in mind a preference relation $>$ and a distinguished alternative $x^{s}$ and judges satisfactory those alternatives that are at least as good as $x^{s}$ and unsatisfactory those that are worse than $x^{s}$. The DM searches through the budget set $A$ according the menu sequence $\mathbf{A}$ and if she identifies at least one satisfactory alternative in the first stage, then she stops searching at $A_{1}$, which becomes the consideration set, and selects the $>$-maximal alternative available in $A_{1}$. Otherwise, she explores the second stage and the procedure is the same as before. If there is no satisfactory alternative in the budget set $A$ (i.e., $U C_{>}\left(A ; x^{s}\right)=\emptyset$ ), then she keeps searching until she discovers the whole budget set. In this case she selects the best unsatisfactory element from $A$. Notice that this procedure is not demanding in terms of memory as it requires the DM to keep in mind just one alternative (the best one) at each stage of the process. For this reason, we believe that it is reasonable to assume that the DM chooses the best unsatisfactory alternative available once she has explored the whole budget set.

\section{Fully Observable Menu Sequences}

Over the last decades various data enrichment techniques have been developed in experimental economics to shed light on the process that leads a DM to make a decision. The mouselab technology, for instance, allows for monitoring which attributes have been looked up in multi-attribute and multi-alternative decision problems. The more sophisticated eye-tracking device records eye-movement images and allows the experimenter to infer subject's information acquisition patterns. Search behavior can be observed and recorded outside the laboratory too. In online purchases, for instance, firms can easily infer which products have been examined before the transaction is completed by looking at the links that the consumer has explored. Another example is the stores in which consumers are forced to follow a given path between the shelves from the entrance to the exit and there are shortcuts for those consumers who stop searching before completing the tour (e.g. IKEA). The choice domain $\mathcal{D}_{1}$, defined in section 2, aims to capture all those situations in which search order is observable. 
Suppose that we observe the DM making choices. Our first concern is to provide a behavioral definition of satisfaction $\left(x^{s}\right)$. Assume that $c$ is an SCF. How can we tell whether an alternative is satisfactory or not? Consider a menu sequence $\langle\{x\}, X\rangle \in$ $\mathcal{D}_{1}$. Since a satisficing DM always stops searching as soon as she identifies the first satisfactory alternative, then $x$ being satisfactory implies that the DM must choose $x$ from $\langle\{x\}, X\rangle$. To see that this condition is also sufficient for $x$ to be satisfactory, assume, by contradiction, that $x$ is chosen from $\langle\{x\}, X\rangle$ and $x$ is unsatisfactory. This implies that the DM considers all alternatives in the grand set and $x$ is the $>$-maximal alternative. However, since there is at least one satisfactory alternative in the grand set, then $x$ has to be satisfactory, which contradicts our previous assumption.

Proposition $1\left(\mathcal{D}_{1}\right.$-Revealed Satisfaction). Assume that the choice function $c$ is an $S C F$. Then, $x \in U C_{>}\left(X ; x^{s}\right)$ if and only if $x=c(\langle\{x\}, X\rangle)$. Whenever this occurs we say that $x$ is $\mathcal{D}_{1}$-revealed to be satisfactory.

Notice that if $\left\{x^{s}\right\}=\max (X ;>)$, then $c$ is rationalizable by a strict linear order. That is, if the aspiration level that partitions the grand set into satisfactory and unsatisfactory alternatives is the best alternative in $X$, then the DM behaves as if she was a maximizer.

We are also interested in investigating whether, given two alternatives $x, y \in X$, $x$ is revealed to be preferred to $y$ or vice versa. Assume that $x=c(\mathbf{A})$ and $y \in A$. This information is not enough for ensuring that $x>y$. To see why, assume that $y$ is preferred to $x, x=c(\mathbf{A})$, and $y \in A$. This does not lead to a contradiction, because the DM might have stopped searching before exploring $y$ and chosen $x$. Therefore, in order to identify the revealed preference, we first need to define behavioral attention.

Let $x \in A$ and define $m(x, \mathbf{A}) \equiv \min \left\{j \mid x \in A_{j}\right\}$ to be the first stage of the menu sequence $\mathbf{A}$ to which $x$ belongs. Assume the DM chooses some alternative $y$ from $\mathbf{A}$ and we are instersted in verifying whether she considers $x \in A$. If $x=y$, then obviously

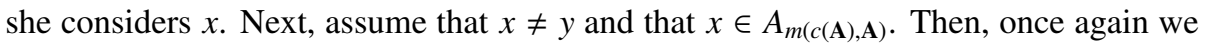
can conclude that the DM considers $x$. The reason is that since $y$ is chosen, then it must be that all alternatives that precede $y$ in the sequence (or are discovered at the same time as $y)$ are considered. Finally, assume that $x \notin A_{m(c(\mathbf{A}), \mathbf{A})}$. This implies that the chosen alternative $y$ precedes $x$ at the menu sequence $\mathbf{A}$. The only way in which the 
DM can explore $x$ is that the chosen alternative $y$ is not a satisfactory alternative. We already know, by proposition 1 , that this occurs whenever $y \neq c(\langle\{y\}, X\rangle)$. Whenever one of these three cases occur we say that $x$ attracts attention at $\mathbf{A}$.

Proposition $2\left(\mathcal{D}_{1}\right.$-Revealed Attention). Suppose that $c$ is an $S C F$ and $x \in A$. Then, $x \in \Gamma_{(\mathbf{A})}$ if and only if one of the following cases occur.

1. $x \in A_{m(c(\mathbf{A}), \mathbf{A})}$ or

2. $y=c(\mathbf{A})$ and $y \neq c(\langle\{y\}, X\rangle)$.

Whenever this occurs we say that $x$ attracts attention at $\mathbf{A}$.

Given $x, y \in X$, we will have that $x$ is revealed to be preferred to $y$ whenever $x$ is chosen, $y$ is available, and $y$ attracts attention.

Proposition $3\left(\mathcal{D}_{1}\right.$-Revealed Preference). Suppose that the choice function $c$ is an SCF. Then, $x>y$ if and only if there is some $\mathbf{A} \in \mathcal{D}_{1}$ such that $x=c(\mathbf{A})$ and $y$ attracts attention at $\mathbf{A}$. Whenever this occurs we say that $x$ is $\mathcal{D}_{1}$-revealed to be preferred to $y$.

We now move to the axiomatic characterization. We propose two properties.

Limited Consideration WARP (LCWARP). Assume that $x$ and $y$ attract attention at $\mathbf{A}$ and $\mathbf{B}$ and $x \neq y$. Then, if $x=c(\mathbf{A})$, then $y \neq c(\mathbf{B})$.

Standard WARP requires that given two alternatives $x, y \in A \cap B$, if $x=c(\mathbf{A})$, then $y \neq c(\mathbf{B})$ without distinguishing between alternatives that the DM considers and alternatives that she does not. LCWARP is a weakening of WARP and requires the extended choice function to satisfy WARP, provided that the DM pays attention to $x$ and $y$ at both choice problems according to the definition provided in proposition $2 .{ }^{4}$

\footnotetext{
${ }^{4}$ Masatlioglu et al. (2009) introduce an axiom called 'WARP with Limited Attention'. The difference between Limited Consideration WARP and WARP with Limited Attention is in the definition of 'paying attention to'. Masatlioglu et al. (2009) define a set to be a consideration set whenever the removal of one alternative that the DM does not consider does not change the consideration set. On the contrary, in this paper the consideration set is defined according to the definition of proposition 2 .
} 
Satisficing (SAT). If $x=c(\langle\{x\}, X\rangle)$, then $x \notin A_{m(c(\mathbf{A}), \mathbf{A})-1}$ for all $\mathbf{A} \in \mathcal{D}_{1}$ such that $x \in A$.

SAT requires that if an alternative is revealed to be satisfactory, then it cannot be the case that the DM discovers $x$ and then explores further the menu sequence. In other words, if $x$ is acceptable, then it cannot happen that the DM discovers and chooses an alternative $y$ at some stage of the menu sequence, given that an earlier stage she explored $x$.

Theorem 1. An extended choice function $c$ is an SCF if and only if c satisfies LCWARP and $S A T .^{5}$

Given a pair $\left(>, x^{s}\right)$, let $c_{\left(>, x^{s}\right)}$ be the SCF induced by $\left(>, x^{s}\right)$.

Proposition 4 (Uniqueness). Let $c$ be an extended choice function on $\mathcal{D}_{1}$. Given two pairs $\left(>, x^{s}\right)$ and $\left(>^{\prime}, x^{s \prime}\right)$, if $\left(>, x^{s}\right) \neq\left(>^{\prime}, x^{s \prime}\right)$, then $c_{\left(>, x^{s}\right)} \neq c_{\left(>^{\prime}, x^{s \prime}\right)} \cdot{ }^{6}$

The results of this subsection allow us to infer non-parametrically whether DM's behavior is consistent with the satisficing procedure simply by testing LCWARP and SAT. In addition, by using propositions 1 and 2 we can infer both satisfaction and preference. In order for our results to hold we need to observe the choices made from following three sets of menu sequences:

1. every A such that $A_{1}=A$ and $|A| \leq 3,{ }^{7}$

2. every $\langle\{x\}, X\rangle$ for any $x \in X,{ }^{8}$ and

3. every A such that $\left|A_{1}\right|=2$ and $|A|=3 .^{9}$

If the grand set contained three alternatives, this would mean that we would need to observe just ten out of twenty-two possible menu sequences.

\footnotetext{
${ }^{5}$ Proofs of the theorem and independence of the axioms are given in the appendix.

${ }^{6}$ The result follows from propositions 1 and 3 .

${ }^{7}$ These observations are needed to construct the strict linear order $>$ and to infer revealed preference.

${ }^{8}$ These observations are needed to infer satisfaction.

${ }^{9}$ These observations are needed in theorem 1 to prove that alternatives that are revealed to be satisfactory are preferred over those unsatisfactory.
} 
We conclude this section by noting that if, on the one hand, the assumption that search order is observable might sound demanding in some circumstances, then, on the other hand, it is also true that under this assumption relatively few observations are needed in order to obtain the above results. Moreover, as proposition 4 suggests, preferences and aspiration level can be uniquely identified.

\section{Partially Observable Menu Sequences}

In this section we assume that for each decision problem we observe the feasible set, the choice made by the DM, and the starting point from which the search process begins. The starting point can be either a single alternative or a menu of alternatives. We can interpret the starting point as a default option or menu, as past consumption, as a social norm or convention, or as a focal point. Imagine a consumer who examines the products displayed on a supermarket shelf. The observable starting point could be the set of items placed at the head of the shelf that the consumer necessarily considers. Those items are very likely to attract attention, because they are placed in a strategic position, they are often advertised and sometimes discounted. Another example could be the 'menu of the day' in a restaurant. Costumers typically first examine the dishes in the menu of the day and then eventually look at the paper-menu.

In order to capture this kind of situations we introduce the domain $\mathcal{D}_{2} \subseteq\left\{\left(A_{1}, A\right) \mid A_{1} \subseteq\right.$ $A \in \mathcal{P}(X)\}$. We interpret $\left(A_{1}, A\right)$ as a choice problem, where $A$ is a budget set and $A_{1}$ is the first (observable) stage of the menu sequence. The remaining stages (if any) are assumed to be unobservable. Let $c$ be the extended choice function defined on $\mathcal{D}_{2}{ }^{10}$

Definition 3. $c$ is a Satisficing Choice Function defined on $\mathcal{D}_{2}\left(S_{C} F_{2}\right)$ if and only if there exist a strict linear order $>$ on $X$ and a distinguished alternative $x^{s} \in X$ such that, for any $\left(A_{1}, A\right) \in \mathcal{D}_{2}$,

$$
\left\{c\left(A_{1}, A\right)\right\} \equiv \max \left(\Gamma_{\left(A_{1}, A\right)} ;>\right)
$$

\footnotetext{
${ }^{10}$ We assume that $c\left(A_{1}, A\right) \in A$ is non-empty and single-valued. At the end of this section we will specify what data we need to observe in order to get our results.
} 
where $\Gamma_{\left(A_{1}, A\right)} \subseteq A$ is a a consideration set mapping

$$
\Gamma_{\left(A_{1}, A\right)} \equiv \begin{cases}A_{1} & \text { if } U C_{>}\left(A_{1} ; x^{s}\right) \neq \emptyset \\ \bar{A} & \text { if } U C_{>}\left(A \backslash A_{1} ; x^{s}\right) \neq \emptyset=U C_{>}\left(A_{1} ; x^{s}\right) \\ A & \text { otherwise }\end{cases}
$$

and $\bar{A}$ is any set such that $A_{1} \subset \bar{A} \subseteq A$.

Our interpretation of the $\mathrm{SCF}_{2}$ is analogous to the SCF's. The only difference is that if the budget set $A$ contains some satisfactory alternatives and these alternatives are not in $A_{1}$, then the consideration set is given by $\bar{A}$, where $\bar{A}$ is some subset of $A$ such that $A_{1} \subset \bar{A} \subseteq A$.

Now we move to the behavioral definitions of satisfaction and preference. The definitions of revealed satisfaction and preference are analogous to the ones of previous section.

Proposition $5\left(\mathcal{D}_{2}\right.$-Revealed Satisfaction). Suppose that $c$ is an $S C F_{2}$. Then, $x \in$ $U C_{>}\left(X ; x^{s}\right)$ if and only if $x=c(\{x\}, X)$. Whenever this occurs we say that $x$ is $\mathcal{D}_{2-}$ revealed to be satisfactory.

Proposition $6\left(\mathcal{D}_{2}\right.$-Revealed Preference). Suppose that the choice function $c$ is an $S C F_{2}$. Then, $x>y$ if and only if there is some $\left(A_{1}, A\right) \in \mathcal{D}_{2}$ such that $x=c\left(A_{1}, A\right)$ and $y \in A_{1}$. Whenever this occurs we say that $x$ is $\mathcal{D}_{2}$-revealed to be preferred to $y$.

Now we move to the axiomatic characterization. We propose two properties that are analogous to the ones illustrated in the previous section: Limited Consideration WARP and SAT under $\mathcal{D}_{2}$ (LCWARP ${ }_{2}$ and $\mathrm{SAT}_{2}$, respectively).

LCWARP $_{2}$ differs from LCWARP in the conditions under which we impose standard WARP to hold. Consider the following definition.

Definition 4. Let $x \in A$. We say that $x$ matters at $\left(A_{1}, A\right)$ whenever one of the following cases occur.

1. $x \in A_{1}$ or $x=c\left(A_{1}, A\right)$.

2. $y=c\left(A_{1}, A\right)$ and $y \neq c(\{y\}, X)$. 
3. $y=c\left(A_{1}, A\right) \notin A_{1}$ and there is no $\left(C_{1}, C\right) \in \mathcal{D}_{2}$ such that $y \in C_{1}$ and $x=c\left(C_{1}, C\right)$.

Here is our interpretation. It is clear that under the domain $\mathcal{D}_{2}$ we can infer attention only partially, because search order is just partially observable. In particular we can infer that $x$ is part of the DM's consideration set only if two cases occur. First, either $x$ belongs to the first stage of the menu sequence or $x$ is chosen (case 1 of definition 4). Second, the chosen alternative is revealed to be unsatisfactory and therefore the DM considers all alternatives in the budget set, including $x$ (case 2). However, these conditions are not necessary for $x$ to be part of the DM's consideration set, because it can happen that the chosen alternative $y$ is satisfactory, $x, y \notin A_{1}$, and $x \in \bar{A}$. In this case $x$ is part of the consideration set. However, since $\bar{A}$ is unobservable, then it is impossible to infer whether this occurs or not. Nevertheless, we can say something about in-attention in this case. Assume that the chosen alternative $y$ is satisfactory and $x, y \notin A_{1}$. Notice that if $x$ is revealed to be preferred to $y$, then it cannot be that $x$ attracts attention at $\left(A_{1}, A\right)$. The reason is that if this was the case, then the DM would have chosen $x$ instead of $y$. Therefore, a necessary condition for $x$ to attract attention at $\left(A_{1}, A\right)$ is that the chosen alternative $y$ has to be revealed to be preferred to $x$ (case $3)$. However, this condition is not sufficient, because it might still be $x$ does not attract attention at $\left(A_{1}, A\right)$.

LCWARP $P_{2}$ Assume that $x$ and $y$ matter at $\left(A_{1}, A\right)$ and $\left(B_{1}, B\right)$. Then, if $x=$ $c\left(A_{1}, A\right)$, then $y \neq c\left(B_{1}, B\right)$.

On the other hand, $\mathrm{SAT}_{2}$ is a straightforward reformulation of SAT under the domain $\mathcal{D}_{2}$.

$\mathbf{S A T}_{2}$. If $x=c(\{x\}, X)$, then $c\left(A_{1}, A\right) \in A_{1}$ for any $\left(A_{1}, A\right) \in \mathcal{D}_{2}$ such that $x \in A_{1}$.

Theorem 2. An extended choice function c is an $\mathrm{SCF}_{2}$ if and only if c satisfies $L C W A R P_{2}$ and $S A T_{2}{ }^{11}$

${ }^{11}$ Since LCWARP and SAT are independent, then it is easy to figure out that also LCWARP 2 and $\mathrm{SAT}_{2}$ 
Given a pair $\left(>, x^{s}\right)$, let $c_{\left(\succ, x^{s}\right)}$ be the $\mathrm{SCF}_{2}$ induced by $\left(>, x^{s}\right)$.

Proposition 7 (Uniqueness). Let $c$ be an extended choice function on $\mathcal{D}_{2}$. Given two pairs $\left(>, x^{s}\right)$ and $\left(>^{\prime}, x^{s \prime}\right)$, if $\left(>, x^{s}\right) \neq\left(>^{\prime}, x^{s \prime}\right)$, then $c_{\left(>, x^{s}\right)} \neq c_{\left(\succ^{\prime}, x^{s}\right)}{ }^{12}$

In order for our results to hold we need to observe the choices made from the following problems:

1. every $\left(A_{1}, A\right)$ such that $A_{1}=A$ and $|A| \leq 3,{ }^{13}$

2. every $(\{x\}, X)$ for any $x \in X,{ }^{14}$ and

3. every $\left(A_{1}, A\right)$ such that $\left|A_{1}\right|=2$ and $|A|=3 .{ }^{15}$

If the grand set $X$ contained 3 alternatives, then we would need ten out of sixteen possible observations.

Interestingly by observing search order just partially, we are still able to characterize the proposed procedure and uniquely identify satisfaction and preference. The only sacrifices are that we can only partially infer attention and the proportion of needed data under $\mathcal{D}_{2}$ is greater than under $\mathcal{D}_{1}$. The latter suggests that in relative terms it is more useful to a policy-maker to have few detailed observations $\left(\mathcal{D}_{1}\right)$ than many coarser ones $\left(\mathcal{D}_{2}\right)$.

\section{Unobservable Menu Sequences}

In this section we assume, as in standard revealed preference theory, that search behavior is not observable. That is, we can observe just the feasible set of alternatives and the choice made by the DM. Imagine a consumer who searches for some product in a marketplace. In this case inferring her search behavior appears a rather difficult task. The reason is that there are many entries to the marketplace and consumers can

\footnotetext{
do. The proof of the theorem is given in the appendix.

${ }^{12}$ The result follows from propositions 5 and 6 .

${ }^{13}$ These observations are needed to construct the strict linear order $>$ and to infer revealed preference.

${ }^{14}$ These observations are needed to infer satisfaction.

${ }^{15}$ These observations are needed in theorem 2 to prove that alternatives that are revealed to be satisfactory are preferred over those unsatisfactory.
} 
freely move from one stand to the other. Another example is those circumstances in which alternatives are available to the DM, but consideration, comparison, and analysis of the various options mainly take place in the DM's mind (e.g. chess).

Formally, let $\mathcal{D}_{3} \subseteq \mathcal{P}(X) .{ }^{16}$ Let $C(A) \subseteq A$ be a choice correspondence defined on $\mathcal{D}_{3}$. We define $C$ to be an Induced Satisficing Choice Correspondence whenever $C$ records the choices made by the DM who follows the satisficing procedure under multiple menu sequences. Under this domain we assume that we can repeatedly observe the DM making choices.

Definition 5. $C$ is an Induced Satisficing Choice Correspondence (ISCC) whenever there exists an $S C F c$ such that, for any $A \in \mathcal{D}_{3}$,

$$
C(A) \equiv\{x \in A \mid x=c(\mathbf{A}) \text { for some menu sequence } \mathbf{A} \in \Sigma(A)\}
$$

We first investigate the conditions under which we can unambiguously state that an alternative is satisfactory. Notice that if an alternative $x$ is acceptable, then, by proposition $1, x$ is chosen from the menu sequence $\langle\{x\}, X\rangle$. If $C$ is an ISCC, then this implies that $x \in C(X)$. In the other direction if $x \in C(X)$, then it must be that $x=c(\mathbf{X})$ for some $\mathbf{X} \in \Sigma(X)$ implying that $x$ is satisfactory.

Proposition $8\left(\mathcal{D}_{3}\right.$-Revealed Satisfaction). Suppose that $C$ is an ISCC. Then, $x \in$ $U C_{>}\left(X ; x^{s}\right)$ if and only if $x \in C(X)$. Whenever this occurs we say that $x$ is $\mathcal{D}_{3}$-revealed to be satisfactory.

Assume that $x \in C(X)$. Let $A \subseteq X$ and $\langle\{x\}, \ldots, A\rangle \in \mathcal{D}_{1}$ be a menu sequence. Since $x \in C(X)$, then, by proposition $8, x$ is satisfactory and, therefore, $x=c(\langle\{x\}, \ldots, A\rangle)$. Assuming that $C$ is an ISCC, this implies that $x \in C(A)$. In other words, a satisfactory alternative is always chosen whenever available. This implies that, under this domain, we cannot infer what is the ranking between satisfactory alternatives. On the contrary, we can recover the preference relation over unsatisfactory alternatives. Assume that $A=\{x, y\}$ and $x, y$ are unsatisfactory. Independently of the menu sequence the DM

\footnotetext{
${ }^{16}$ At the end of this section we will specify what data we need for our results to hold.
} 
pays attention to both alternatives. Therefore, if she prefers $x$ to $y$, then $x=c(\mathbf{A})$ for any A. This implies that $\{x\}=C(A)$. On the contrary, if she prefers $y$ to $x$, then $\{y\}=C(A)$.

Proposition $9\left(\mathcal{D}_{3}\right.$-Revealed Preference). Let $C$ be an ISCC and $x, y \notin U C_{\succ}\left(X ; x^{s}\right)$. Then, $x>y$ if and only if there is some $A \in \mathcal{P}(X)$ such that $\{x\}=C(A)$ and $y \in A$. Whenever this occurs we say that $x$ is $\mathcal{D}_{3}$-revealed to be preferred to $y$.

Now we move to the axiomatic characterization.

We propose two properties. The first one is the well-known Weak Axiom of Revealed Preference.

Weak Axiom of Revealed Preference (WARP). Given $x, y \in A \cap B$, if $x \in C(A)$ and $y \in C(B)$, then $x \in C(B)$.

The second one is called Maximal Indifference and requires that if an alternative $x$ is jointly chosen with another alternative $y$, then $x$ has to be chosen from the grand set.

Maximal Indifference (MI). If $x, y \in C(A)$, then $x \in C(X)$.

Here is our interpretation. Assume that a satisficing DM is confronted with a budget set $A$. If there is no satisfactory alternative in $A$, then independently of the menu sequence, she will always choose the same alternative, say $x$. This implies that the induced choice correspondence is a singleton $\{x\}=C(A)$. Hence, for $C(A)$ not to be a singleton, it must be that there are at least two satisfactory alternatives in $A$. Assume that $x, y \in C(A)$. By the above argument, this implies that both $x$ and $y$ are acceptable alternatives. MI simply requires that since $x, y \in C(A)$ reveals that $x$ is satisfactory, then $x$ has to be chosen from the grand set $X$. Too see why this axiom is reasonable, assume that it is violated. That is, $x$ is revealed to be satisfactory and is not chosen from the grand set $X$. Since $x \notin C(X)$, then this implies that there is no menu sequence $\mathbf{X}$ such that $x=c(\mathbf{X})$. This sounds to be an implausible conclusion as we know that a satisficing DM always stops searching as soon as she identifies the first acceptable alternative and there are menu sequences in $\mathcal{D}_{1}$ such that $x$ is the first satisfactory 
alternative available. ${ }^{17}$

Theorem 3. $C$ is an ISCC if and only if it satisfies WARP and MI. ${ }^{18}$

Given a pair $\left(>, x^{s}\right)$, let $C_{\left(>, x^{s}\right)}$ be the ISCC induced by $\left(>, x^{s}\right)$. Next, let $>_{\text {unsat }} \subseteq>$ be the preference relation $>$ restricted to unsatisfactory alternatives only.

As far as uniqueness is concerned, notice that given two pairs $\left(>, x^{s}\right)$ and $\left(>^{\prime}, x^{s \prime}\right)$, $\left(>, x^{s}\right) \neq\left(\succ^{\prime}, x^{s \prime}\right)$ implies that $C_{\left(\succ, x^{s}\right)} \neq C_{\left(\succ^{\prime}, x^{\prime \prime}\right)}$ if and only if either $x^{s} \neq x^{s \prime}$ or $>_{\text {unsat }} \neq>_{\text {unsat }}^{\prime}$ or both. The reason is that under the domain $\mathcal{D}_{3}$ we can infer preference only below the aspiration level. This implies that the identification process is unique only under the above conditions.

In order for our results to hold we need to observe the choices made from the following budget sets.

1. every $A$ such that $|A| \leq 3,{ }^{19}$ and

2. the grand set $X .^{20}$

In addition, we need that whenever an alternative $x$ is revealed to be satisfactory, then the DM chooses from a menu sequence $\mathbf{B}$ that lists $x$ before any other satisfactory alternative in $\mathbf{B}$ for any $B \in \mathcal{D}_{3}$ such that $x \in B$ and $\left|U C_{>}\left(B ; x^{s}\right)\right|>1$. We come back to this assumption in a paragraph below. Let $X=\{x, y, z\}$ and assume that $x$ and $y$ are satisfactory. Then, we need four out of four possible observations, which are $c(\{x, y\})$, $c(\{x, z\}), c(\{y, z\})$, and $c(X)$, and that the DM explores first $x$ (resp., $y)$ and then $y$ (resp., $x)$ at both $X$ and $\{x, y\}$.

We draw several conclusions from these results. First, by assuming that search order is unobservable, we are still able to infer satisfaction and preference but only below the threshold. Moreover, we need to make minimal assumptions about what are the menu sequences that the DM is required to choose from. However, on the other hand, the proportion of needed data is higher than under the first two informational cases

\footnotetext{
${ }^{17}$ E.g. all menu sequences such that $\langle\{x\}, \ldots\rangle$.

${ }^{18}$ Proofs of the theorem and independence of the axioms are given in the appendix.

${ }^{19}$ These observations are needed to infer preference.

${ }^{20}$ This observation is needed to infer satisfaction.
} 
suggesting that there is a tradeoff going on: on the one hand, observing more variables allows for recovering more information about the DM and relative less observations are needed. On the other hand, observing less variables implies a limited inference of the DM's characteristics and relatively more observations are needed.

Second, standard WARP is often violated in the real world. However, we propose a characterization, which is a special case of the standard model. Indeed, $C$ is not rationalizable by any weak order, but only by the weak order that admits indifference only among maximal alternatives. A similar result is established by Rubinstein and Salant (2006). The intuition behind our result is that in order for theorem 3 to hold we need to assume that the satisficing DM chooses from some key menu sequences. If this assumption holds, then the induced choice correspondence is more than rational for the following reason. First, if a budget set $A$ does not contain any satisfactory alternative (or just one), then the DM always chooses the same alternative independently of the menu sequence. Second, if $A$ contains more than one satisfactory element, then there is always a menu sequence that lists a satisfactory alternative before any other satisfactory alternative implying that the DM chooses it. This implies in turn that the induced choice correspondence will include all satisfactory alternatives available in $A$ for any $A$ and place them at the top of the ranking. On the other hand, without the above assumption, the choice correspondence induced by an SCF may be very irrational. Assume that $x \in C(A), y \in C(B)$, and $x, y \in A \cap B$. This choice pattern implies that $x$ and $y$ are satisfactory. If the DM does not choose from a menu sequence $\mathbf{B}$ that lists $x$ before $y$, then we will never observe $x \in C(B)$. Hence, we would not be able to show that $C$ satisfies WARP. The conclusion is that there might be satisficing DMs that violate WARP out there. A reason for which we observe violations may be because they do not choose from some key menu sequences.

Third, it is easy to see that if we define the induced choice correspondence as $C_{P}(A) \equiv\left\{x \in A \mid x=c\left(A_{1}, A\right)\right.$ for some $\left.A_{1} \subseteq A\right\}$ with $c$ being an $\mathrm{SCF}_{2}$, we get exactly the same results. The reason is that under the domain $\mathcal{D}_{3}$ by assuming that $C$ is an ISCC we can infer satisfaction and preference over unsatisfactory alternatives. Since this information can be recovered also under the domain $\mathcal{D}_{2}$, then, by defining the induced choice correspondence as a $C_{P}$, we do not sacrifice anything. 


\section{Relationships with Other Models}

\subsection{Choice from Lists}

Rubinstein and Salant (2006) propose a model in which the DM chooses from lists of alternatives. Formally, given any two stages $A_{j}, A_{j+1}$ of a menu sequence $\mathbf{A}, \mathbf{A}$ is defined to be linear whenever $\left|A_{j}\right|=\left|A_{j+1}\right|-1$ for all $j=1, \ldots, N_{\mathbf{A}}-1$. Let $\mathcal{D}_{L} \subset \mathcal{D}_{1}$ be the domain $\mathcal{D}_{1}$, where all menu sequences in $\mathcal{D}_{1}$ are linear. A Rubinstein and Salant's list is a linear menu sequence.

Rubinstein and Salant (2006) characterize the set of choice functions that maximize some preference relation, where indifference is resolved according to the position that alternatives take up in the lists: either the first or the last maximal alternative is selected. Formally, let $\geq=>\cup \sim$ be a weak order on $X$ and let $\delta: X \rightarrow\{1,2\}$ be a priority indicator, where $>$ and $\sim$ denote the asymmetric and symmetric parts of $\geq$, respectively. ${ }^{21}$ Let $\delta(x)=\delta(y)$ whenever $x \sim y$. Given any list $\mathbf{A} \in \mathcal{D}_{L}$, let $D_{\geq, \delta}$ be a choice function that chooses from $A$ either the first or the last $\geq$-maximal element of the list $\mathbf{A}$ depending on whether the $\delta$-value of the $\geq$-maximal set is 1 or 2 , respectively.

Proposition 10. Let $c$ be an extended choice function defined on $\mathcal{D}_{L}$. If $c$ is an $S C F$, then it is also a $D_{\geq, \delta}$, but the converse is not true.

We omit the proof of this proposition as in the appendix we generalize Rubinstein and Salant (2006)'s model to menu sequences and then show that our model is a special case of theirs by providing an intuition of our results.

The procedure proposed by Rubinstein and Salant (2006) encompasses a more general class of models than ours. Nevertheless, there are several aspects of our model that makes it complementary to theirs.

First, we generalize lists to menu sequences. That is, we do not assume that the DM necessarily examines alternatives one by one. As we largely discussed in section 2 , there are many real world examples that fit within our model, but do not within theirs.

\footnotetext{
${ }^{21} \mathrm{~A}$ weak order is a complete and transitive binary relation. Let $>\equiv\{(x, y) \in X \times X \mid(x, y) \in \geq$ and $(y, x) \notin \geq\}$ be its asymmetric and $\sim \equiv\{(x, y) \in X \times X \mid(x, y) \in \geq$ and $(y, x) \in \geq\}$ its symmetric part.
} 
Second, unlike Rubinstein and Salant (2006), our model allows for inferring preferences above the threshold $x^{s}$. To see why their model does not, notice that a satisficing DM always stops searching as soon as she identifies the first satisfactory alternative. Since according to Rubinstein and Salant (2006)'s domain the DM examines alternatives one by one, then it is impossible to infer the ranking between satisfactory alternatives.

\subsection{Choice with Frames}

Salant and Rubinstein (2008) propose a framework called choice with frames according to which the choice function depends not only on the budget set, but also on some observable information, called frame, which is irrelevant from a standard viewpoint. Formally, a choice problem with frames is a pair $(A, f)$, where $A \in \mathcal{P}(X)$ is a budget set and $f \in F$ is a frame in the set of frames. The first two versions of our model $\left(\mathrm{SCF}\right.$ and $\mathrm{SCF}_{2}$ ) can also be defined in terms of choices with frames. We first examine the SCF.

Let $O$ be a weak ordering on $X$, representing an ordering with which the DM examines alternatives. We identify the choice function with frames $c(A, O)$ with the extended choice function $c(O \mid A)$, where $O \mid A$ is the menu sequence induced by restricting the order $O$ to a budget set $A$. An SCF, which we denote by $c_{S C F}(O \mid A)$, can then be defined as:

$$
c_{S C F}(O \mid A) \equiv \max \left(A ;>_{O}\right)
$$

where $>_{O}$ depends on the frame $O$ and is constructed as follows.

$$
\begin{aligned}
>_{O} \equiv & \left(>\backslash\left\{(x, y) \in>\mid x, y \in U C_{>}\left(X ; x^{s}\right)\right\}\right) \\
& \cup\left\{(x, y) \in>\mid x, y \in U C_{>}\left(X ; x^{s}\right) \text { s.t. } x O y \text { and }(\neg(y O x) \text { or } x>y)\right\}
\end{aligned}
$$

Each $>_{O}$ modifies the ranking between satisfactory alternatives depending on what is the positioning that alternatives take up in the ordering $O$. Given a frame $O$ and two satisfactory alternatives $x$ and $y$, if $x$ strictly $O$-precedes $y$, then independently of whether $x>y$ or $y>x$ we have that $x>_{O} y$. The reason is that, under the frame $O$, the 
DM always discovers $x$ before discovering $y$. Since both $x$ and $y$ are satisfactory, then the DM always stops searching as soon as she discovers $x$ and chooses it. On the other hand, if $x O$-precedes $y$ (weakly) and $x>y$, then $>_{O}$ coincides with $>$. Finally, notice that the ranking between unsatisfactory alternatives is not affected.

This definition suggests that any $c_{S C F}(O \mid A)$ satisfies WARP. In Salant and Rubinstein (2008)'s terms we would say that $c_{S C F}(O \mid A)$ is an example of a salient consideration function. ${ }^{22}$ Interestingly, this implies that violations of WARP can take place only when the DM mixes two or more frames $O$. Since the proposed model does not place any restriction on the way in which the DM discovers alternatives, then it is straightforward that an SCF can explain a variety of irrational choice patterns, such as cycles. ${ }^{23}$ Nevertheless, it is not true that the SCF can explain any choice pattern. ${ }^{24}$

Now we move to analysis of the $\mathrm{SCF}_{2}$. It turns out that the $\mathrm{SCF}_{2}$ is not a salient consideration function. Too see why, notice that, given a problem $\left(A_{1}, A\right) \in \mathcal{D}_{2}$ and fixing the 'frame' $A_{1}$, an $\mathrm{SCF}_{2}$ admits the choice reversal phenomenon. Let $z, w$ be two unsatisfactory and $x, y$ two satisfactory alternatives. Suppose that alternatives are disposed on a circular shelf (see figure 1) and that the DM' starting point is the singleton $\{z\}$.

Assume for the moment that product $w$ is unavailable and that the DM decides to explore the alternatives clockwise. In this case she discovers $x$, which is the first satisfactory alternative available, and, therefore, stops searching, and chooses it. Next, assume that $w$ becomes now available and the DM decides to explore the alternatives anti-clockwise. In this case, she first discover $w$, an unsatisfactory alternative, and continues her search process until she reaches $y$. Since $y$ is satisfactory, then she stops searching and selects it. In sum, $x=c(\{z\},\{x, y, z\})$ and $y=c(\{z\},\{x, y, z, w\})$, which is

\footnotetext{
${ }^{22}$ An extended choice function $c$ is a Salient Consideration function if for every frame $f \in F$, there exists a corresponding ordering $\rangle_{f}$ such that $c(A, f)$ is the $>_{f}$ - maximal element in A (Salant and Rubinstein, 2008).

${ }^{23}$ Let $\langle\{x\},\{x, y\}\rangle,\langle\{y\},\{y, z\}\rangle,\langle\{z\},\{x, z\}\rangle \in \mathcal{D}_{1}$ and assume that $x, y, z$ are satisfactory. Then, $x=$ $c(\langle\{x\},\{x, y\}\rangle), y=c(\langle\{x\},\{x, y\}\rangle)$, and $z=c(\langle\{x\},\{x, y\}\rangle)$, which is clearly a cyclical choice pattern.

${ }^{24}$ Assume that $x \in U C_{>}\left(X ; x^{s}\right)$. Let $\langle\{x\}, \ldots\rangle \in \mathcal{D}_{1}$ be a menu sequence. Since $x$ is satisfactory, then the DM must stop searching at the first stage and choose $x$. Therefore, any $c$ that does not select $x$ from $\langle\{x\}, \ldots\rangle$ is not an SCF.
} 


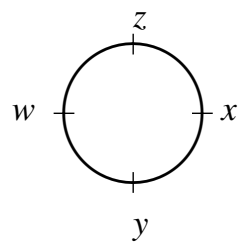

Figure 1: A circular shelf.

not compatible with the definition of salient consideration function.

\subsection{Agenda Rationalizability}

Apesteguia and Ballester (2010) propose a model in which the alternatives in $X$ are ordered according to some linear order $<$. Let $T$ be a tournament and let $\mathbf{A}_{>}$be a list induced by ordering the alternatives in $A \subseteq X$ according to $<{ }^{25}$ Given any list $A_{<}$, the DM compares the first two alternatives in the list and selects the winner according to $T$. Then she compares the selected item with the third alternative in the list and again selects the winner according to $T$. The DM proceeds in this way until she compares the surviving alternative with the last element of the list. The winner of this last comparison constitutes the final choice $c\left(\mathbf{A}_{<}\right)$. Let $e\left(T, \mathbf{A}_{<}\right)$denote the chosen alternative from a list $\mathbf{A}_{<}$according to the above procedure and whenever $e\left(T, \mathbf{A}_{<}\right)=c\left(\mathbf{A}_{<}\right)$we say that $c$ is agenda rationalizable.

Proposition 11. If $c$ is an $S C F$, then it is agenda rationalizable, but the converse is not true.

In the agenda model the DM examines always alternatives according to $<$. As we know from the previous section, if a satisficing DM explores alternatives according to the same ordering, then an SCF is rationalizable by a strict linear order. This implies that an SCF is agenda rationalizable. However, the converse is not true, because a

\footnotetext{
${ }^{25} \mathrm{~A}$ tournament is an asymmetric and complete binary relation.
} 
tournament might generate cyclical choice patterns that are not consistent with the definition of SCF.

\subsection{Choice by Iterative Search}

Masatlioglu and Nakajima (2009) propose a choice procedure, called choice by iterative search (CIS), in which the DM starts by exploring an exogenously given contemplation point and considers only those alternatives that are similar to it. If the contemplation point is the best alternative in the consideration set, then the DM stops searching and chooses it. Otherwise, she keeps searching, the best alternative available becomes the new contemplation point, and the procedure is the same as before. This process continues until the the contemplation point is the best alternative in the consideration set. Masatlioglu and Nakajima (2009) define their model on the domain $\mathcal{D}_{S P} \subseteq\{(\{x\}, A) \mid A \in \mathcal{P}(X)$ and $x \in A\}$. Details of the Masatlioglu and Nakajima (2009)'s model and proof can be found in the appendix.

Proposition 12. Let $c$ be an extended choice function defined on $\mathcal{D}_{S P}$. If $c$ is an $S C F_{2}$, then it is also a CIS, but the converse is not true.

The intuition behind this result can be figured out by looking at the CIS revealed preference relation. Masatlioglu and Nakajima (2009) argue that $x$ is revealed to be preferred to $y$ whenever they observe either (i) $x=c(\{y\}, A)$ or (ii) $x=c(\{z\}, A)$ and $z=c(\{y\}, B)$. That is, (i) $x$ is chosen when $y$ is the starting point or (ii) $x$ is indirectly revealed to be preferred to $y$. Notice that if $c$ was an $\mathrm{SCF}_{2}$, then we would reach exactly the same conclusion. However, in our model there are other circumstances in which we can conclude that $x$ is preferred over $y$. Suppose, for instance, that $x=c(\{z\}, A)$, $y \in A$, and $z \neq c(\{z\}, X)$. This choice pattern reveals that since $z$ is unsatisfactory, then all alternatives in $A$ are considered by the DM, $x$ is the most preferred alternative in $A$, and, therefore, $x$ is preferred to $y$. Notice that in the CIS model this does not apply. In sum, under the domain $\mathcal{D}_{S P}$, the satisficing revealed preference relation (strictly) includes the CIS one. 


\subsection{Choice with Initial Endowments}

Masatlioglu and Ok (2009) propose a model of choice in which the DM deals with choice problems both with and without initial endowment. In particular, if she faces choice problems without an initial endowment, she chooses by maximizing some utility function $U$, as in the standard model. On the other hand, whenever she deals with choice problems with an initial endowment, she follows a two-stage procedure. In the first stage, she restricts her attention to a subset of the available alternatives. This set includes all elements that are superior to the initial endowment in an unambiguous sense. If $x \in X$ is an initial endowment, then we denote this set by $Q(x)$, where $x \in$ $Q(x)$. Then, in the second stage, she chooses from the restricted set by maximizing her utility function. That is, given a budget set $A$ and an initial endowment $x \in A$, the DM chooses $\arg \max _{y \in A \cap Q(x)} U(y){ }^{26}$

In our framework each $(\{x\}, A) \in \mathcal{D}_{S P}$ can be thought as a choice problem with an initial endowment.

Proposition 13. Let $c$ be an extended choice function defined on $\mathcal{D}_{S P}$. If $c$ is a choice with initial endowments, then it is not necessarily an $\mathrm{SCF}_{2}$ and also the converse is true.

We prove this by means of two examples. Consider first the following scenario: $x=c(\{y\}, A), y=c(\{w\}, B)$, and $x \in B$. This choice pattern clearly violates LCWARP $_{2}$. The reason is that $x=c(\{y\}, A)$ reveals that $x>y$ and $y$ is unsatisfactory. Since $y=c(\{w\}, B)$, then the DM considers all alternatives in $B$ (including $x)$ revealing that $y>x$, a contradiction. However, this choice pattern is compatible with a choice with initial endowments. To see why, notice that in reference-dependent preference models, such as Masatlioglu and Ok (2009), preferences change with respect to the reference point. Therefore, in the previous example it might be case that $x \in Q(y)$, but $x \notin Q(w)$. That is, $x$ is unambiguously better than $y$, when $y$ is the starting point, but does not

\footnotetext{
${ }^{26}$ See also Masatlioglu and Ok (2005) and Apesteguia and Ballester (2009) as other studies that investigate status-quo biased and reference-dependent behavior.
} 
clearly dominate $w$, when $w$ is the initial endowment. Therefore, $x=c(\{y\}, A)$ and $y=c(\{w\}, B)$ with $x$ being available are not contradictory.

In the other direction, assume that $x=c(\{z\}, A), y=c(\{z\}, B), x=c(\{x\}, X), y=$ $c(\{y\}, X)$, and $x, y \in B \subset A$. Notice first that this choice pattern is rationalizable by an $\mathrm{SCF}_{2}$. Let $x \in \Gamma_{(\{z\}, A)}, y \in \Gamma_{(\{z\}, B)}, y \notin \Gamma_{(\{z\}, A)}$, and $x \notin \Gamma_{(\{z\}, B)}$. That is, assume that the DM pays attention to $x$ at $(\{z\}, A)$ and to $y$ at $(\{z\}, B)$ and does not consider $y$ at $(\{z\}, A)$ and $x$ at $(\{z\}, B)$. This assumption is feasible, because $x=c(\{x\}, X)$ and $y=c(\{y\}, X)$ reveal that both $x$ and $y$ are satisfactory. Hence, the satisficing DM simply chooses the maximal alternative from the consideration set, which is $x$ at $(\{z\}, A)$ and $y$ at $(\{z\}, B)$. On the other hand, this choice pattern is not compatible with a choice with initial endowments. The reason is that since $x=c(\{z\}, A)$, then this implies that $x$ is unambiguously better than $z$, when $z$ is the reference point. Since, $y=c(\{z\}, B)$, then the same applies to $y$. However, since $x, y \in B \subset A$, then we infer that $\{x, y\} \subseteq Q(z) \cap A \cap B$. Given that the DM chooses the alternative with the highest utility from the sets $Q(z) \cap A$ and $Q(z) \cap B$, then $x=c(\{z\}, A)$ and $y=c(\{z\}, B)$ imply that $x$ yields more utility than $y$ and $y$ yields more utility than $x$, respectively, which is clearly a contradiction.

\section{Concluding Remarks}

This paper proposes a theory of boundedly rational choice within the revealed preference approach in which DM's behavior is consistent with the satisficing heuristic.

Our main contribution is that we investigate behavioral definitions of satisfaction and preference and axiomatically characterize the proposed model under three different choice domains. This allows us to figure out the extent to which by expanding the informational structure we can gain a deeper understanding of the proposed model. We have found that the revealed information is monotonic. That is, the more detailed the observations, the more we can infer about the DM's behavior. However, at the same time, as observations become less detailed, we need relatively more data in order to recover the primitive elements of the model.

Over the last few years various models of boundedly rational choice related to satisficing have been proposed. We devote a section of our paper to analyze the formal 
connections between our framework and most of those models. ${ }^{27}$

Another interesting feature of our model is that it is easy to use in applications. Consider the following example. Assume that $n \geq 2$ firms compete in order to maximize market shares and each firm produces one good and has to decide its quality. The higher the quality of a good, the higher its production cost. Suppose that firms face a population of 'satisficing' consumers. That is, each of them discovers and analyzes alternatives sequentially and stops searching as soon as she identifies the first satisfactory alternative, where each item's positioning in the DM's preference ranking depends on its quality. An interesting and policy-relevant research question could be to investigate firms' equilibrium behavior under the assumption that firms do not know with certainty what are consumers' aspiration levels.

A non-trivial extension of our work could be to assume that the threshold is not fixed, but can vary depending on the characteristics of the choice problem, such as complexity. We leave this for future research.

\section{Acknoledgements}

This paper is based on a chapter of my PhD dissertation. I would like to thank Jose Apesteguia, Miguel A. Ballester, Barton Lipman, Paola Manzini, Marco Mariotti, Ricardo A. Marques Pereira, Yusufcan Masatlioglu, Luigi Mittone, William Neilson, Nicholas C. Yannelis, and two anonymous referees for their helpful suggestions. I am also grateful to the seminar audience at the universities Autonoma de Barcelona, Pompeu Fabra, St.Andrews, and Trento and the participants at the 11th SAET meeting (2011, Faro) and EEA-ESEM conference (2011, Oslo) for their interesting comments. Financial support from the Scottish Institute for Economic Research is gratefully acknowledged. Any error is my own responsibility.

\footnotetext{
${ }^{27}$ Apesteguia and Ballester (2010) perform a similar exercise in their choice by sequential procedures model.
} 


\section{References}

Apesteguia, J., Ballester, M. A., 2009. A theory of reference-dependent behavior. Eonomic Theory 40, 427-455.

Apesteguia, J., Ballester, M. A., 2010. Choice by sequential procedures. Universitat Pompeu Fabra and Universitat Autonoma de Barcelona. Working Paper.

Caplin, A., Dean, M., 2011. Search, choice, and revealed preference. Theoretical Economics 6, 19-48.

Caplin, A., Dean, M., Martin, D., 2011. Search and satisficing. American Economic Review 101 (7), 2899-2922.

Eliaz, K., Richter, M., Rubinstein, A., 2011. Choosing the two finalists. Economic Theory 46, 211-219.

Eliaz, K., Spiegler, R., 2011. Consideration sets and competitive marketing. Review of Economic Studies 78, 235-262.

Horan, S., 2010. Sequential search and choice from lists. Boston University. Job Market Paper.

Manzini, P., Mariotti, M., 2007. Sequentially rationalizable choice. American Economic Review 97 (5), 1824-1839.

Mas-Colell, A., Whinston, M. D., Green, J. R., 1995. Microeconomic Theory. New York: Oxford University Press.

Masatlioglu, Y., Nakajima, D., 2009. Choice by iterative search. Department of Economics, University of Michigan. Working Paper.

Masatlioglu, Y., Nakajima, D., Ozbay, E. Y., 2009. Revealed attention. American Economic Review (forthcoming).

Masatlioglu, Y., Ok, E. A., 2005. Rational choice with status-quo bias. Journal of Economic Theory 121, 1-29. 
Masatlioglu, Y., Ok, E. A., 2009. A canonical model of choice with initial endowment. Department of Economics, University of Michigan and New York University. Working Paper.

Raymond, C., 2011. Revealed search theory. University of Michigan. Working Paper.

Reutskaja, E., Nagel, R., Camerer, C. F., Rangel, A., 2011. Search dynamics in consumer choice under time pressure: An eye-tracking study. American Economic Review 101, 900-926.

Richter, M. K., 1966. Revealed preference theory. Econometrica 34 (3), 635-645.

Rubinstein, A., Salant, Y., 2006. A model of choice from lists. Theoretical Economics $1(1), 3-17$.

Salant, Y., Rubinstein, A., 2008. $(a, f)$ : Choices with frames. Review of Economic Studies 75, 1287-1296, issue 4.

Sen, A. K., 1971. Choice functions and revealed preference. The Review of Economic Studies 38 (3), 307-317.

Simon, H. A., 1955. A behavioral model of rational choice. The Quarterly Journal of Economics 69 (1), 99-118.

Tyson, C. J., 2008. Cognitive constraints, contraction consistency, and the satisficing criterion. Journal of Economic Theory, 138, 51-70.

\section{Appendix A. Relationships with Other Models}

Appendix A.1. Choice from Lists

Rubinstein and Salant (2006) show that an axiom called List Independence of Irrelevant Alternatives is necessary and sufficient for the choice function from lists to be 
a $D_{\geq, \delta} \cdot{ }^{28}$

List Independence of Irrelevant Alternatives (LIIA): if $x=c(\mathbf{A})$, then $x=c(\mathbf{A} \backslash$ $\{y\})$ for any $y \in A \backslash\{x\}$.

That is, assume that an alternative $x$ is chosen. Then, removing any alternative $y \neq x$ from the list does not change the choice, ceteris paribus.

We first generalize Rubinstein and Salant (2006)'s result to menu sequences and then show that our procedure is a special case of their model. Given a strict linear order $P$ and a weak order $\geq=>\cup$, we say that $P$ is based on $\geq$ whenever two cases occur: (i) $x>y>z$ implies $x P z$ and (ii) $x>y$ implies $x P y$ whenever there exists an alternative $z$ such that either $x \sim z$ or $y \sim z$.

Let $\geq$ be a weak order on $X$ and $\delta$ a priority indicator as defined in Rubinstein and Salant (2006). Let $P$ be a strict linear order on $X$ based on $\geq$. We introduce the choice function $D_{\geq, P, \delta}$, according to which the DM stops searching as soon as she identifies the first or the last $\geq$-maximal alternative in the menu sequence depending on whether the $\delta$-value of the $\geq$-maximal set is equal to 1 or to 2 , respectively. Let the set of discovered alternatives be denoted by $A_{j}$. Then, the DM selects the $P$-maximal alternative from the set $A_{j} \backslash A_{j-1}$.

Definition 6. $c$ is a $D_{\geq, P, \delta}$ whenever there exist a unique weak order $\geq$, a unique strict linear order $P$, and a unique priority indicator $\delta$ such that

$$
\{c(\mathbf{A})\} \equiv \begin{cases}\max \left(A_{m} \backslash A_{m-1} ; P\right) & \text { if } \delta(z)=1 \\ \max \left(A_{M} \backslash A_{M-1} ; P\right) & \text { if } \delta(z)=2\end{cases}
$$

where (i) $m \equiv \min \left\{j \mid A_{j} \cap \max (A ; \geq) \neq \emptyset\right\}$,

$$
\text { (ii) } M \equiv \max \left\{j \mid \emptyset \neq A_{j} \cap \max (A ; \geq) \neq A_{j-1} \cap \max (A ; \geq)\right\} \text {, }
$$

\footnotetext{
${ }^{28}$ Rubinstein and Salant (2006) show that List Independence of Irrelevant Alternatives is equivalent to another axiom called Partition Independence according to which the DM chooses the same alternative from a list independently of whether she chooses from the list or she partitions the list into two sublists, chooses from each sublist, and finally makes a choice between the two chosen element from the sublists.
} 
(iii) $P$ is based on $\geq$, and

(iv) $z \in \max (A ; \geq)$.

Under the domain $\mathcal{D}_{L}$ alternatives can be pairwise compared in two ways: either $x$ precedes $y$ or $y$ precedes $x$. Rubinstein and Salant (2006) define the weak order $\geq$ on these binary comparisons and use LIIA to derive their procedure. On the contrary, under the larger domain $\mathcal{D}_{1}$ a third type of binary comparison between alternatives is possible: $x$ and $y$ are discovered at the same time by the DM. We define the binary relation $P$ on the latter and, by using LIIA, we extend Rubinstein and Salant (2006)'s procedure. This is the intuition at the basis of theorem 4, which states that LIIA is a necessary and sufficient condition for $c$ to be a $D_{\geq, P, \delta}$.

Theorem 4. $c$ is a $D_{\geq, P, \delta}$ if and only if c satisfies LIIA.

Notice that under the domain $\mathcal{D}_{L}$ every set $A_{j} \backslash A_{j-1}$ is a singleton and $D_{\succeq, P, \delta}$ reduces to $D_{\geq, \delta}$.

Now we investigate the relationships between $D_{\geq, P, \delta}$ and our model. Consider the following property.

Perfect Attention Position Independence (PAPI): if $x=c(\mathbf{A})$ and $x \neq c(\langle\{x\}, X\rangle)$, then $x=c(\mathbf{B})$ for any $\mathbf{B} \in \mathcal{D}_{1}$ such that $A=B$.

PAPI requires that if an alternative $x$ is chosen and revealed to be unsatisfactory at some menu sequence $\mathbf{A}$, then $x$ has to be chosen from any other menu sequence $\mathbf{B}$ such that $A=B$. That is, if $x$ is chosen and revealed to be unacceptable at $\mathbf{A}$, then this implies that all alternatives in $A$ attract attention at $\mathbf{A}$. Then the axiom requires that $x$ has to be chosen no matter what is its positioning in the sequence. It turns out that LIIA and PAPI are logically equivalent to LCWARP and SAT, as the next proposition illustrates. ${ }^{29}$

\footnotetext{
${ }^{29}$ In appendix A we show that LIIA and PAPI are independent.
} 
Proposition 14. The following statements are equivalent.

1. c satisfies LCWARP and SAT.

2. c satisfies LIIA and PAPI.

3. $c$ is an $S C F$.

Rubinstein and Salant (2006) propose a procedure that includes the satisficing heuristic as a special case. In their paper they offer the following example. Let $c$ be a $D_{\geq, \delta}$, assume that $\geq$ has two indifference classes, where the $\delta$-value of the maximal set is equal to 1 and the $\delta$-value of the minimal one is equal to 2. A DM following this procedure stops searching as soon as she identifies the first satisfactory alternative. On the other hand, if there is no satisfactory alternative available, then she selects the last unsatisfactory alternative in the list. Interestingly, an additional interpretation emerges from our analysis. Assume that $c$ is a $D_{\geq, \delta}$, let $\delta(z)=1$ for all $z \in X$, and assume that all indifference classes apart from the $\geq$-maximal one are singletons. Set $\max (X ; \geq)$ to be the set of satisfactory alternatives and the strict part of $\geq$ to be the strict linear order that ranks unsatisfactory alternatives. Moreover, rank the satisfactory alternatives according to any ordering. It is easy to see that the resulting choice function is an SCF.

Corollary 1. Let c be an extended choice function.

1. If $c$ is an $S C F$, then $c$ is a $D_{\geq, \delta}$.

2. If $c$ is $D_{\geq, \delta}$, then $c$ is an SCF if and only if the following holds.

(a) all indifference classes apart from the $\geq$-maximal one are singletons;

(b) the $\delta$-value is equal to 1 for all alternatives in the grand set.

\section{Appendix A.2. Relationships with Choice by Iterative Search}

In Masatlioglu and Nakajima (2009) a choice problem is a pair $(\{x\}, A)$, where $A$ is a budget set and $x \in A$ a feasible contemplation point. Let $>$ be a strict linear order representing DM's preferences and let $\Omega(x, A) \subseteq A$ be a consideration set mapping. Masatlioglu and Nakajima (2009) distinguish between context-dependent and context-free consideration sets. The former is the most general version of their model and implies that $\Omega(\cdot)$ depends on both the contemplation point and the budget set in 
an arbitrary manner. The latter implies that whether an alternative is considered is independent of the other alternatives. A Choice by Iterative Search $(C I S)$ is a choice function $c$ defined on $\mathcal{D}_{S P}=\{(\{x\}, A) \mid A \in \mathcal{P}(X)$ and $x \in A\}$ such that there exists a strict linear order $>$, a consideration set mapping $\Omega$, and, for every choice problem $\left(\left\{x_{0}\right\}, A\right)$, a sequence of contemplation points $\left(x_{0}, x_{1}, \ldots, x_{n}, y\right)$ such that (i) $x_{k}$ is the $>$-maximal element in $\Omega\left(x_{k-1}, A\right)$ for $k=1, \ldots, n$ and (ii) $y=c\left(\left\{x_{0}\right\}, A\right)$ is $>$-maximal in both $\Omega\left(x_{n}, A\right)$ and $\Omega(y, A){ }^{30}$

Masatlioglu and Nakajima (2009) show that CIS with context-dependent consideration sets is equivalent to the following two properties.

Dominating Anchor Axiom: for any $B \in \mathcal{P}(X)$ there exists $x^{*} \in B$ such that

(i) $x^{*}=c\left(\left\{x^{*}\right\}, A\right)$ whenever $A \subseteq B$

(ii) $c\left(\left\{x^{*}\right\}, A\right) \notin B \backslash\left\{x^{*}\right\}$ otherwise.

Anchor Bias Axiom: if $y=c(\{x\}, A)$, then $y=c(\{y\}, A)$.

The dominating anchor axiom requires that for every budget set $A$ there exists an alternatives $x^{*}$ that blocks all the other alternatives $y$ in $A \backslash\left\{x^{*}\right\}$. That is, $y$ cannot be chosen whenever $x^{*}$ is the starting point. On the other hand, the anchor bias axiom requires that if some alternative $y$ is chosen when $x$ is the starting point, then $y$ has to be chosen also when $y$ itself is the contemplation point.

It turns out that if $c$ is a CIS with context-dependent consideration sets, then it is also an $\mathrm{SCF}_{2}$, but the converse is not true. ${ }^{31}$

\section{Appendix B. Independence of the Axioms}

LCWARP does not imply SAT. Let $X=\{x, y, z\} . x=c(\langle\{x\},\{x, y\}\rangle), x=c(\langle\{x\},\{x, z\}\rangle)$, $x=c(\langle\{y\},\{x, y\}\rangle), z=c(\langle\{y\},\{y, z\}\rangle), z=c(\langle\{z\},\{x, z\}\rangle), z=c(\langle\{z\},\{y, z\}\rangle), x=$

\footnotetext{
${ }^{30}$ Masatlioglu and Nakajima (2009) assume that $c(\{x\}, A) \in A$ is non-empty and single-valued.

${ }^{31}$ It can be shown that (i) if $c$ is a CIS with context-free consideration sets, then $c$ is not necessarily $\mathrm{SCF}_{2}$ and (ii) also the converse is true.
} 
$c(\langle\{x\},\{x, y\}, X\rangle), x=c(\langle\{x\},\{x, z\}, X\rangle), x=c(\langle\{y\},\{x, y\}, X\rangle), z=c(\langle\{y\},\{y, z\}, X\rangle)$, $z=c(\langle\{z\},\{x, z\}, X\rangle), z=c(\langle\{z\},\{y, z\}, X\rangle), z=c(\langle\{x, y\}, X\rangle), z=c(\langle\{x, z\}, X\rangle), z=$ $c(\langle\{y, z\}, X\rangle), x=c(\langle\{x\}, X\rangle), z=c(\langle\{y\}, X\rangle), z=c(\langle\{z\}, X\rangle), x=c(\langle\{x, y\}\rangle), z=$ $c(\langle\{x, z\}\rangle), z=c(\langle\{y, z\}\rangle)$, and $z=c(\langle X\rangle)$. This extended choice function satisfies LCWARP, but violates SAT.

SAT does not imply LCWARP. Let $X=\{x, y, z\} . x=c(\langle\{x\},\{x, y\}\rangle), x=c(\langle\{x\},\{x, z\}\rangle)$, $x=c(\langle\{y\},\{x, y\}\rangle), z=c(\langle\{y\},\{y, z\}\rangle), z=c(\langle\{z\},\{x, z\}\rangle), z=c(\langle\{z\},\{y, z\}\rangle), x=$ $c(\langle\{x\},\{x, y\}, X\rangle), x=c(\langle\{x\},\{x, z\}, X\rangle), x=c(\langle\{y\},\{x, y\}, X\rangle), z=c(\langle\{y\},\{y, z\}, X\rangle)$, $z=c(\langle\{z\},\{x, z\}, X\rangle), z=c(\langle\{z\},\{y, z\}, X\rangle), x=c(\langle\{x, y\}, X\rangle), z=c(\langle\{x, z\}, X\rangle), y=$ $c(\langle\{y, z\}, X\rangle), x=c(\langle\{x\}, X\rangle), z=c(\langle\{y\}, X\rangle), z=c(\langle\{z\}, X\rangle), x=c(\langle\{x, y\}\rangle), z=$ $c(\langle\{x, z\}\rangle), y=c(\langle\{y, z\}\rangle)$, and $x=c(\langle X\rangle)$. This extended choice function satisfies SAT, but violates LCWARP.

WARP does not imply MI. Let $X=\{x, y, z\} .\{x\}=C(\{x, y, z\}),\{x\}=C(\{x, y\})$, $\{x\}=C(\{x, z\})$, and $\{y, z\}=C(\{y, z\})$. This choice correspondence satisfies WARP, but violates MI.

MI does not imply WARP. Let $X=\{x, y, z\} .\{x\}=C(\{x, y, z\}),\{y\}=C(\{x, y\})$, $\{x\}=C(\{x, z\})$, and $\{y\}=C(\{y, z\})$. This choice correspondence satisfies MI, but violates WARP.

LIIA does not imply PAPI. Let $X=\{x, y, z\}$. Let $c$ be a $D_{\succeq, P, \delta}$ and assume that $x \sim_{2} y>z$ and $x P y P z$. This choice function satisfies LIIA, but violates PAPI

PAPI does not imply LIIA. Let $X=\{x, y, z\}$. Choose $x$ at all choice problems of cardinality 3, $y$ at all choice problems of cardinality 2 to which $y$ belongs, and $x$ at the remaining choice problems of cardinality 2 . This choice function satisfies PAPI, but 
violates LIIA

\section{Appendix C. Proofs of Propositions}

Proof of Proposition 12. Let $c$ be an extended choice function defined on $\mathcal{D}_{S P}$.

Assume that $c$ is an $\mathrm{SCF}_{2}$. We want to show that it is also a CIS with contextdependent consideration sets. We first show that $c$ satisfies the Dominating Anchor Axiom.

Assume that $x=c(\{x\}, A)$. Assume first that $x$ is satisfactory. This implies that $x=c(\{x\}, B)$, independently of whether or not $B$ is a subset of $A$. Next, suppose that $x$ is unsatisfactory. This implies that $x$ is $>$-maximal in $A$. Therefore, $x=c(\{x\}, B)$ for any $B \subseteq A$. In addition, $c(\{x\}, B) \notin A \backslash\{x\}$, which is the desired result.

Now we show that $c$ satisfies also the Anchor Bias Axiom. Assume that $y=$ $c(\{x\}, A)$. Suppose first that $y$ is satisfactory. This immediately implies that $y=$ $c(\{y\}, A)$. Next, suppose that $y$ is unsatisfactory. This implies that $y$ is maximal in $A$, there are no satisfactory alternatives in $A$, and $y$ is chosen from $A$ independently of its positioning. Hence, $y=c(\{y\}, A)$.

To see that the converse is not true, consider the following example. Assume that $x=c(\{y\}, A), y=c(\{z\}, B)$, and $x \in B$. Notice that whereas the Dominating Anchor and the Anchor Bias Axioms are satisfied, $\mathrm{LCWARP}_{2}$ is not

Proof of Proposition 14. We just need to show is that LCWARP and SAT are logically equivalent to LIIA and PAPI, because we already know from theorem 1 that LCWARP and SAT are necessary and sufficient for $c$ to be an SCF.

Necessity. Assume that $c$ satisfies LCWARP and SAT.

We first show that $c$ satisfies LIIA. Let $x=c(\mathbf{A})$ and let $\Gamma_{(\mathbf{A})}$ be the set of alternatives that attract attention at $\mathbf{A}$. Since $c$ is an SCF, then $x$ is $>$-maximal in $\Gamma_{(\mathbf{A})}$ and, therefore, $x=c(\mathbf{A} \backslash\{y\})$ for any $y \in \Gamma_{(\mathbf{A})}$. Next, if $\Gamma_{(\mathbf{A})} \subset A$, then $x$ is satisfactory and removing any $y \in A \backslash \Gamma_{(\mathbf{A})}$ does not affect choices. Hence, $x=c(\mathbf{A} \backslash\{y\})$ also for any $y \in A \backslash \Gamma_{(\mathbf{A})}$. 
Now we show that $\mathrm{c}$ satisfies PAPI. Let $x=c(\mathbf{A})$ and $x \neq c(\langle\{x\}, X\rangle)$. This implies that $x$ is unsatisfactory and all alternatives in $A$ attract attention at A. Since $c$ is an SCF, then $x$ is $>$-maximal in $A$. Hence, $x=c(\mathbf{B})$ for any $\mathbf{B} \in \mathcal{D}_{1}$ such that $B=A$, which is the desired result.

Sufficiency. Assume that $c$ satisfies LIIA and PAPI. Suppose, by contradiction, that $c$ violates either LCWARP or SAT or both. We distinguish three cases.

Case (i): $c$ satisfies SAT, but violates LCWARP. In particular, assume that $x, y$ attract attention at $\mathbf{A}$ and at $\mathbf{B}$ and that $x=c(\mathbf{A})$ and $y=c(\mathbf{B})$. Then there are two sub-cases.

Sub-case (a): $x$ and $y$ are revealed to be satisfactory. This implies that necessarily $y \in A_{m(c(\mathbf{A}), \mathbf{A})} \backslash A_{m(c(\mathbf{A}), \mathbf{A})-1}$ and $x \in B_{m(c(\mathbf{B}), \mathbf{B})} \backslash B_{m(c(\mathbf{B}), \mathbf{B})-1}$. By repeatedly applying LIIA to $x=c(\mathbf{A})$ and $y=c(\mathbf{B})$, we get $x=c(\langle\{x, y\}\rangle)$ and $y=c(\mathbf{C})$, where $\mathbf{C} \backslash\{z\}=\langle\{x, y\}\rangle$, which contradicts LIIA.

Sub-case (b): either $x$ or $y$ or both are revealed to be unsatisfactory. Assume WLOG $x$ is revealed to be satisfactory and $y$ is not. Since $x=c(\mathbf{A})$ and $y$ attracts attention at $\mathbf{A}$, then either $y \in A_{m(c(\mathbf{A}), \mathbf{A})} \backslash A_{m(c(\mathbf{A}), \mathbf{A})-1}$ or $y \in A_{m(c(\mathbf{A}), \mathbf{A})-1}$. Assume WLOG that $y \in$ $A_{m(c(\mathbf{A}), \mathbf{A})-1}$. By repeatedly applying LIIA, we get $x=c(\langle\{y\},\{x, y\}\rangle)$. Since $y=c(\mathbf{B})$ and $y$ is revealed to be unsatisfactory, then, by PAPI, there exists a choice problem $\mathbf{C}$ such that $y \in C_{j}, x \in C_{j+1} \backslash C_{j}, y=c(\mathbf{C})$, and $C=B$. By repeatedly applying LIIA, we get $y=c(\langle\{y\},\{x, y\}\rangle)$, which leads to a contradiction.

Case (ii): $c$ satisfies LCWARP, but violates SAT. Assume that $x=c(\langle\{x\}, X\rangle)$ and there is some $\mathbf{A} \in \mathcal{D}_{1}$ such that $x \in A_{m(c(\mathbf{A}), \mathbf{A})-1}$. Let $y=c(\mathbf{A})$. Since $x=c(\langle\{x\}, X\rangle)$ and $y=c(\mathbf{A})$, then, by repeatedly applying LIIA, we get $x=c(\langle\{x\},\{x, y\}\rangle)$ and $y=$ $c(\langle\{x\},\{x, y\}\rangle)$, respectively, which leads to a contradiction.

Case (iii) $c$ violates LCWARP and SAT. A contradiction takes place from what we proved in cases (i) and (ii) 


\section{Appendix D. Proofs of Theorems}

Proof of Theorem 1. It is easy to check necessity.

Sufficiency. Assume that $c$ satisfies LCWARP and SAT.

STEP 1: There exists a strict linear order $>$ on $X$, where $x>y$ whenever $x=c(\mathbf{A})$ and $A=A_{1}=\{x, y\}$.

Proof. Let $x>y$ whenever $x=c(\mathbf{A})$, where $A=A_{1}=\{x, y\}$. Notice that $y$ attracts attention at $\mathbf{A}$. It is immediate to see that $>$ is asymmetric, irreflexive, and complete. Next, we show that $>$ is transitive. Assume that $x>y$ and $y>z$. Consider a $\mathbf{C} \in \mathcal{D}_{1}$ such that $C=C_{1}=\{x, y, z\}$. Since $C_{1}=C$, then necessarily $x, y$, and $z$ attract attention at $\mathbf{C}$. Notice that if $z=c(\mathbf{C})$, then, by LCWARP, $z>y$, which leads to a contradiction. Similarly, it cannot be that $y=c(\mathbf{C})$. Therefore, $x=c(\mathbf{C})$. Then, by LCWARP, $x>z$. Hence, $>$ is transitive. Therefore, we conclude that $>$ is a strict linear order. $\square$

STEP 2: Let $\mathbf{A} \in \mathcal{D}_{1}$ and assume that $\Gamma_{(\mathbf{A})} \subseteq A$ is the set of alternatives that attract attention at $\mathbf{A}$. Then, $\{c(\mathbf{A})\}=\max \left(\Gamma_{(\mathbf{A})} ;>\right)$.

Proof. Let $\mathbf{A} \in \mathcal{D}_{1}$ and assume that $\Gamma_{(\mathbf{A})} \subseteq A$ is the set of alternatives that attract attention at $\mathbf{A}$. Let $x=c(\mathbf{A})$. Suppose, by contradiction, that $y>x$ for some $y \in$ $\Gamma_{(\mathbf{A})} \backslash\{x\}$. In this case, $y=c(\mathbf{B})$, where $B=B_{1}=\{x, y\}$. Since $x$ and $y$ attract attention at $\mathbf{A}$, then, by LCWARP, $x \neq c(\mathbf{A})$, which leads to a contradiction. Therefore, there are no $y \in \Gamma_{(\mathbf{A})} \backslash\{x\}$ such that $y>x$. This implies that $\{c(\mathbf{A})\}=\max \left(\Gamma_{(\mathbf{A})} ;>\right)$, which is the desired result.

STEP 3: There exists a partition $(S, X \backslash S)$ of the grand set $X$ such that $x>y$ for any $x \in S$ and $y \in X \backslash S$, where $S=\{x \in X \mid x=c(\langle\{x\}, X\rangle)\}$.

Proof. Let $S=\{x \in X \mid x=c(\langle\{x\}, X\rangle)\}$. Consider any $\mathbf{A} \in \mathcal{D}_{1}$ such that $w \in$ $A \cap S \neq \emptyset$. Suppose, by contradiction, that $y=c(\mathbf{A}) \notin S$ and let $x \in A \cap S$. Since $y \notin S$, 
then all alternatives in $A$ attract attention at A. By STEP 2, $y>x$. Next, since $y \notin S$, then $y \neq c(\langle\{y\}, X\rangle)$. Let $z=c(\langle\{y\}, X\rangle)$. Notice that $y$ attracts attention at $\langle\{y\}, X\rangle$ and, therefore, by STEP 2, $z>y$. Consider the menu sequence $\mathbf{C}=\langle\{x, y\},\{x, y, z\}\rangle$. We first show that $\mathbf{C}$ is different from $\mathbf{A}$. Suppose, by contradiction, that $z \in A$. Since all alternatives in $A$ attract attention at $\mathbf{A}$, then, by STEP 2, $y>z$, a contradiction. Hence, $\mathbf{C} \neq \mathbf{A}$.

In order to get the desired result we have to show that any choice from $\mathbf{C}$ leads to a contradiction. Assume first that $x=c(\mathbf{C})$. In this case, by STEP 2, $x>y$, which leads to a contradiction. Next, assume $y=c(\mathbf{C})$. This implies that all alternatives attract attention at $\mathbf{C}$ and, therefore, by STEP 2, $y>z$, which leads to a contradiction. Assume then that $z=c(\mathbf{C})$. However, this violates SAT. Since $\{c()\}$ cannot be empty, then $c(\mathbf{A}) \in S$. This implies that $x>y$, which is the desired result. $\square$

Let $x^{s}$ be the $>$-minimal alternative in $S$.

STEP 4: For any $\mathbf{A} \in \mathcal{D}_{1}$,

$$
\Gamma_{(\mathbf{A})}= \begin{cases}A_{\min \left\{j \mid A_{j} \cap S \neq \emptyset\right\}} & \text { if } A \cap S \neq \emptyset \\ A & \text { otherwise }\end{cases}
$$

where $\Gamma_{(\mathbf{A})}$ is the set of alternatives that attract attention at $\mathbf{A}$.

Proof. By STEP 2, $\{c(\mathbf{A})\}=\max \left(\Gamma_{(\mathbf{A})} ;>\right)$, where $\Gamma_{(\mathbf{A})}$ is the set of alternatives that attract attention at $\mathbf{A}$. Suppose first that $A \cap S \neq \emptyset$. We have to show that $\Gamma_{(\mathbf{A})}=$ $A_{\min \left\{j \mid A_{j} \cap S \neq \emptyset\right\}}$. Assume first that $x \in \Gamma_{(\mathbf{A})}$. Since $A \cap S \neq \emptyset$, then, by STEP 3 , it must be that $c(\mathbf{A}) \in S$. If this is the case, then $x \in \Gamma_{(\mathbf{A})}$ implies that $x \in A_{m(c(\mathbf{A}), \mathbf{A}) \text {. Since }}$ $c(\mathbf{A}) \in S$, then, by SAT, $c(\mathbf{A}) \in A_{\min \left\{j \mid A_{j} \cap S \neq \emptyset\right\}}$. This implies that also $x \in A_{\min \left\{j \mid A_{j} \cap S \neq \emptyset\right\}}$. Conversely, assume that $x \in A_{\min \left\{j \mid A_{j} \cap S \neq \emptyset\right\}}$. Since $c(\mathbf{A}) \in S$, then $x \in \Gamma_{(\mathbf{A})}$, as desired.

Next, we have to show that $\Gamma_{\mathbf{A}}=A$, whenever $A \cap S=\emptyset$. It is obvious that $x \in \Gamma_{(\mathbf{A})}$ implies $x \in A$. Conversely, assume that $x \in A$. Since $A \cap S=\emptyset$, then all alternatives in $A$ attract attention at $\mathbf{A}$. Hence, $x \in \Gamma_{(\mathbf{A})}$, which is the desired result. $\square$

Therefore, we conclude that $c$ is an SCF. 
Proof of Theorem 2. It is easy to check necessity.

Sufficiency. Assume that $c$ satisfies LCWARP 2 and $\mathrm{SAT}_{2}$. STEP 1, STEP 2, and STEP 3 are analogous to those of Theorem 1. For this reason we omit the proof.

STEP 1: There exists a strict linear order $>$ on $X$, where $x>y$ whenever $x=$ $c\left(A_{1}, A\right)$, where $A_{1}=\{x, y\}=A$.

STEP 2: Let $\left(A_{1}, A\right) \in \mathcal{D}_{2}$ and assume that $\Gamma_{\left(A_{1}, A\right)} \subseteq A$ is the set of alternatives that matter at $\left(A_{1}, A\right)$. Then, $\left\{c\left(A_{1}, A\right)\right\}=\max \left(\Gamma_{\left(A_{1}, A\right)} ;>\right)$.

STEP 3: There exists a partition $(S, X \backslash S)$ of the grand set $X$ such that $x>y$ for any $x \in S$ and $y \in X \backslash S$, where $S=\{x \in X \mid x=c(\{x\}, X)\}$.

STEP 4: For any $\left(A_{1}, A\right) \in \mathcal{D}_{2}$,

$$
\Gamma_{\left(A_{1}, A\right)}= \begin{cases}A_{1} & \text { if } A_{1} \cap S \neq \emptyset \\ \bar{A} & \text { if }\left(A \backslash A_{1}\right) \cap S \neq \emptyset=A_{1} \cap S \\ A & \text { otherwise }\end{cases}
$$

where $\bar{A}=A_{1} \cup\left\{x \in A \backslash A_{1} \mid c\left(A_{1}, A\right)>x\right\} \cup\left\{c\left(A_{1}, A\right)\right\}$ and $\Gamma_{\left(A_{1}, A\right)}$ is the set of alternatives that matter at $\left(A_{1}, A\right)$.

Proof. By STEP 2, $\left\{c\left(A_{1}, A\right)\right\}=\max \left(\Gamma_{\left(A_{1}\right), A} ;>\right)$, where $\Gamma_{\left(A_{1}, A\right)}$ is the set of alternatives that matter at $\left(A_{1}, A\right)$. Suppose first that $A_{1} \cap S \neq \emptyset$. We have to show that $\Gamma_{\left(A_{1}, A\right)}=A_{1}$. Assume first that $x \in \Gamma_{\left(A_{1}, A\right)}$. Since $A_{1} \cap S \neq \emptyset$, then, by STEP 3 , it must be that $c\left(A_{1}, A\right) \in S$. Moreover, by $\mathrm{SAT}_{2}, c\left(A_{1}, A\right) \in A_{1}$. This implies that case 2 and case 3 of definition 4 cannot occur. Hence, only case 1 of definition 4 can occur, that is, $x \in A_{1}$, as desired. Conversely, assume that $x \in A_{1}$. This immediately implies that $x \in \Gamma_{\left(A_{1}, A\right)}$.

Assume that $\left(A \backslash A_{1}\right) \cap S \neq \emptyset=A_{1} \cap S$. We have to show that $\Gamma_{\left(A_{1}, A\right)}=\bar{A}$, where $\bar{A}=A_{1} \cup\left\{x \in A \backslash A_{1} \mid c\left(A_{1}, A\right)>x\right\} \cup\left\{c\left(A_{1}, A\right)\right\}$. Assume first that $x \in \Gamma_{\left(A_{1}, A\right)}$. Since $\left(A \backslash A_{1}\right) \cap S \neq \emptyset=A_{1} \cap S$, then, by STEP $2, c\left(A_{1}, A\right) \in S$, which rules out case 2 of definition 4. This implies that $x \in A_{1}$ (case 1) and $x \in A \backslash A_{1}$ only if $c\left(A_{1}, A\right)>x$ (case 
3) or $x=c\left(A_{1}, A\right)$. Hence, $x \in \bar{A}$. Conversely, assume that $x \in \bar{A}$. Since, by STEP 3 , it must be that $c\left(A_{1}, A\right) \in S$, then $c\left(A_{1}, A\right) \notin A_{1}$. This does not rule out any case of definition 4. Hence, $x \in \Gamma_{\left(A_{1}, A\right)}$, as desired.

Finally, assume that $A \cap S=\emptyset$. We have to show that $\Gamma_{\left(A_{1}, A\right)}=A$. Clearly, $x \in \Gamma\left(A_{1}, A\right)$ implies $x \in A$. Conversely, assume that $x \in A$. Since $A \cap S=\emptyset$, then $c(A) \neq c(\{c(A)\}, X)$. This implies that $x \in \Gamma\left(A_{1}, A\right)$ as desired.

Therefore, we conclude that $c$ is an $\mathrm{SCF}_{2}$

Proof of Theorem 3. Necessity. Assume that $C$ is an ISCC. We have to show that $C$ satisfies WARP and MI. We first show that it satisfies WARP.

Let $x, y \in A \cap B$ and assume that $x \in C(A)$ and $y \in C(B)$. We have to show that $x \in C(B)$. The fact that $x \in C(A)$ and $y \in C(B)$ implies that there exist two menu sequences $\mathbf{A}, \mathbf{B} \in \mathcal{D}_{1}$ such that $x=c(\mathbf{A})$ and $y=c(\mathbf{B})$. Notice that it cannot be that neither (i) both $x$ and $y$ are unsatisfactory, nor (ii) WLOG $x$ is satisfactory and $y$ is not. To see it, notice that (i) if both $x$ and $y$ were unsatisfactory, then the DM would pay attention to all alternatives at $\mathbf{A}$ and $\mathbf{B}$. This would imply that both $x>y$ and $y>x$, a contradiction. Moreover, (ii) if WLOG $x$ was satisfactory and $y$ was not, then the DM would pay attention to all alternatives at $\mathbf{B}$, revealing $y>x$. However, this would contradict the fact that $x$ is satisfactory and $y$ is not. Hence, the only possibility is that both $x$ and $y$ are satisfactory. This implies that $x=c(\langle\{x\}, X\rangle)$ and $y=c(\langle\{y\}, X\rangle)$. Let $\mathbf{B}^{\prime}$ be a menu sequence such that $\mathbf{B}^{\prime}=\langle\{x\}, \ldots, B\rangle$. By SAT, $x=c\left(\mathbf{B}^{\prime}\right)$. Hence, $x \in C(B)$, as desired.

Now we show that $C$ satisfies MI. Assume that $x, y \in C(A)$. We have to show that $x \in C(X)$. The fact that $x, y \in C(A)$ implies that there exist two menu sequences $\mathbf{A}, \mathbf{A}^{\prime} \in \mathcal{D}_{1}$ such that $x=c(\mathbf{A})$ and $y=c\left(\mathbf{A}^{\prime}\right)$. Notice that this can happen if and only if both $x$ and $y$ are satisfactory. But, if this is the case, then $x=c(\langle\{x\}, X\rangle)$ and $y=c(\langle\{y\}, X\rangle)$, which implies that $x \in C(X)$, as desired.

Sufficiency. Assume that $C$ satisfies WARP and MI. We have to show that there 
exists an SCF such that, for any $A \in \mathcal{D}_{3}$,

$$
C(A)=\{x \in A \mid x=c(\mathbf{A}) \text { for some menu sequence } \mathbf{A} \text { of } A\}
$$

It is well-known that if $\mathrm{C}$ satisfies WARP, then there exists a weak order $\geq^{*}$ such that $C(A)=\max \left(A ; \geq^{*}\right)$ for any $A \in \mathcal{P}(X)$, provided that $\mathcal{P}(X)$ contains all subsets of $X$ up to three elements. Next, notice that, by MI, indifference is admitted only among $\geq^{*}$-maximal alternatives. Suppose not and assume, by contradiction, that $x \sim^{*} y$ and there is some $z$ such that $z>^{*} x$. Hence, there exist $A, B \in \mathcal{D}_{3}$ such that $x, y \in C(A)$, $z \in C(B)$, and $x \notin C(B)$. By MI, $x \in C(X)$. Since $z \in X$, then $x \geq^{*} z$, which leads to a contradiction.

Let $c$ be an SCF, where $U C_{>}\left(X ; x^{s}\right) \equiv \max \left(X ; \geq^{*}\right)$ and $>$ is any completion of $>^{*}$. Let $C_{c}(A)=\{x \in A \mid x=c(\mathbf{A})$ for some menu sequence $\mathbf{A}$ of $A\}$. We need to show that $C(A)=C_{c}(A)$. Assume first that $x \in C(A)$. This implies that $x$ is either satisfactory or unsatisfactory and >-maximal in $A$. In either case $x=c(\langle\{x\}, \ldots, A\rangle)$ and, therefore, $x \in C_{c}(A)$. Conversely, if $x \notin C(A)$, then $x$ is a dominated unsatisfactory alternative and there is no menu sequence $\mathbf{A}$ such that $x=c(\mathbf{A})$, which implies that $x \notin C_{c}(A)$, as desired.

Proof of Theorem 4. It is easy to check necessity.

Sufficiency. Assume that $c$ satisfies LIIA. We want to show that $c$ is a $D_{\geq, P, \delta}$. By Proposition 2 in Rubinstein and Salant (2006) there exists a weak order $\geq=>\cup \sim$, such that

- $x>y$ whenever $x=c(\langle\{x\},\{x, y\}\rangle)$ and $x=c(\langle\{y\},\{x, y\}\rangle)$

- $x \sim_{1} y$ whenever $x=c(\langle\{x\},\{x, y\}\rangle)$ and $y=c(\langle\{y\},\{x, y\}\rangle)$

- $x \sim_{2} y$ whenever $y=c(\langle\{x\},\{x, y\}\rangle)$ and $x=c(\langle\{y\},\{x, y\}\rangle)$,

where if $x \sim y$ then either $x \sim_{1} y$ or $x \sim_{2} y$. Moreover, every indifference set $I$ is characterized by the fact that its members relate to each other either by $\sim_{1}$ or by $\sim_{2}$. 
If all alternatives in $I$ are related by $\sim_{1}$, then let $\delta(x)=1$ for all $x \in I$. Otherwise, let $\delta(x)=2$.

Define $P$ as $x P y$ whenever $x=c(\langle\{x, y\}\rangle)$. Clearly, $P$ is irreflexive, complete, and asymmetric. To see that it is also transitive, assume that $x P y$ and $y P z$. This implies that $x=c(\langle\{x, y\}\rangle)$ and $y=c(\langle\{y, z\}\rangle)$. Suppose, by contradiction, that $z P x$. If this is the case, then $z=c(\langle\{x, z\}\rangle)$. Consider the menu sequence $(\langle\{x, y, z\}\rangle)$. Assume that $x=c(\langle\{x, y, z\}\rangle)$. By LIIA, $x=c(\langle\{x, z\}\rangle)$, which contradicts $z P x$. Next, assume $y=c(\langle\{x, y, z\}\rangle)$. By LIIA, $y=c(\langle\{x, y\}\rangle)$, which contradicts $x P y$. Finally, assume that $z=c(\langle\{x, y, z\}\rangle)$. By LIIA, $z=c(\langle\{y, z\}\rangle)$, which contradicts $y P z$. Since $c()$ cannot be empty, then $x>z$. Hence, $>$ is transitive and, therefore, a strict linear order.

We have to show that

$$
\{c(\mathbf{A})\}= \begin{cases}\max \left(A_{m} \backslash A_{m-1} ; P\right) & \text { if } \delta(z)=1 \\ \max \left(A_{M} \backslash A_{M-1} ; P\right) & \text { if } \delta(z)=2\end{cases}
$$

where $m=\min \left\{j \mid A_{j} \cap \max (A ; \geq) \neq \emptyset\right\}, M=\max \left\{j \mid \emptyset \neq A_{j} \cap \max (A ; \geq) \neq A_{j-1} \cap\right.$ $\max (A ; \geq)\}, P$ is based on $\geq$, and $z \in \max (A ; \geq)$. We prove it in several steps.

STEP 1: $P$ is based on $\geq$.

Proof. Assume that $x>y>z$. We want to show that $x P z$. Consider the menu sequence $(\langle\{y\},\{x, y, z\}\rangle)$. Assume first that $y=c(\langle\{y\},\{x, y, z\}\rangle)$. By LIIA, $y=c(\langle\{y\},\{x, y\}\rangle)$, which contradicts $x>y$. Next, assume that $z=c(\langle\{y\},\{x, y, z\}\rangle)$. By LIIA, $z=$ $c(\langle\{y\},\{z, y\}\rangle)$, which contradicts $y>z$. Hence, $x=c(\langle\{y\},\{x, y, z\}\rangle)$. By LIIA, $x=$ $c(\langle\{x, z\}\rangle)$, which implies that $x P z$, as desired.

Next, assume that $x>y$ and there exists an alternative $z$ such that either $x \sim z$ or $y \sim z$. We want to show that $x P y$. Assume first that $x \sim z$ and WLOG $x \sim_{2} z$. By Proposition 2 in Rubinstein and Salant (2006), $z>y$. Consider the menu sequence $(\langle\{z\},\{x, y, z\}\rangle)$. Assume that $z=c(\langle\{z\},\{x, y, z\}\rangle)$. By LIIA, $z=c(\langle\{z\},\{x, z\}\rangle)$, which contradicts the fact that $x \sim_{2} z$. Next, suppose that $y=c(\langle\{z\},\{x, y, z\}\rangle)$. By LIIA, $y=c(\langle\{z\},\{y, z\}\rangle)$, which contradicts $z>y$. Hence, $x=c(\langle\{z\},\{x, y, z\}\rangle)$. By LIIA, $x=c(\langle\{x, y\}\rangle)$, which implies that $x P y$. Assume now that that $y \sim z$ and WLOG $x \sim_{1} z$. 
By Proposition 2 in Rubinstein and Salant (2006), $x>z$. Consider the menu sequence $(\langle\{z\},\{x, y, z\}\rangle)$. Assume that $z=c(\langle\{z\},\{x, y, z\}\rangle)$. By LIIA, $z=c(\langle\{z\},\{x, z\}\rangle)$, which contradicts the fact that $x>z$. Next, suppose that $y=c(\langle\{z\},\{x, y, z\}\rangle)$. By LIIA, $y=c(\langle\{z\},\{y, z\}\rangle)$, which contradicts the fact that $y \sim_{1} z$. Hence, $x=c(\langle\{z\},\{x, y, z\}\rangle)$. By LIIA, $x=c(\langle\{x, y\}\rangle)$, which implies that $x P y$, as desired. $\square$

STEP 2: $c(\mathbf{A}) \in A_{j} \cap \max (A ; \geq) \neq \emptyset$ for some $j$.

Proof. Let $x=c(\mathbf{A})$. Suppose, by contradiction, that $x \in A_{i}$, where $A_{i} \cap \max (A ; \geq$ ) $=\emptyset$. This implies that there exists an alternative $y \in A$ such that $y \in A_{j} \backslash A_{i}$ and $y>x$, where necessarily $j>i$. Since $x=c(\mathbf{A})$, then, by LIIA, $x=c(\langle\{x\},\{x, y\}\rangle)$, which contradicts the fact that $y>x$. Hence, $c(\mathbf{A}) \in A_{j}$ such that $A_{j} \cap \max (A ; \geq) \neq \emptyset$. $\square$

STEP 3: Let $z \in \max (A ; \geq)$.

(i) If $\delta(z)=1$, then, $c(\mathbf{A}) \in A_{m} \backslash A_{m-1}$, where $m=\min \left\{j \mid A_{j} \cap \max (A ; \geq) \neq \emptyset\right\}$.

(ii) If $\delta(z)=2$, then $c(\mathbf{A}) \in A_{M} \backslash A_{M-1}$, where $M=\max \left\{j \mid \emptyset \neq A_{j} \cap \max (A ; \geq) \neq\right.$ $\left.A_{j-1} \cap \max (A ; \geq)\right\}$.

Proof. Let $x=c(\mathbf{A})$. Assume that $\delta(z)=1$, where $z \in \max (A ; \geq)$. Suppose, by contradiction, that $x \notin A_{m}$, where $m=\min \left\{j \mid A_{j} \cap \max (A ; \geq) \neq \emptyset\right\}$. Let $y \in$ $A_{m} \cap \max (A ; \geq)$. Assume first that $x \in A_{m+k} \backslash A_{m}$, where $k>0$. By STEP $2, x \in A_{m+k} \cap$ $\max (A ; \geq) \neq \emptyset$. By LIIA, $x=c(\langle\{y\},\{x, y\}\rangle)$. Then there are two cases. First, assume that $x \in \max (A ; \geq)$. However, this contradicts the fact that $y \sim_{1} x$. Second, assume that $x \notin \max (A ; \geq)$. In this case, $y>x$, which contradicts $x=c(\langle\{y\},\{x, y\}\rangle)$. Next, assume that $x \in A_{m} \cap A_{m-1}$. By LIIA, $x=c(\langle\{y\},\{x, y\}\rangle)$. It cannot be that $x \in \max (A ; \geq)$, because otherwise $A_{m-1}=A_{m}$, which is a contradiction. If $x \notin \max (A ; \geq)$, then $y>x$, which contradicts $x=c(\langle\{y\},\{x, y\}\rangle)$. Hence, $x \in A_{m} \backslash A_{m-1}$.

Part (ii) is proved in a similar way.

STEP 4: Let $z \in \max (A ; \geq)$.

(i) If $\delta(z)=1$, then $\{c(\mathbf{A})\}=\max \left(A_{m} \backslash A_{m-1} ; P\right)$.

(ii) If $\delta(z)=2$, then $\{c(\mathbf{A})\}=\max \left(A_{M} \backslash A_{M-1} ; P\right)$. 
Proof. Let $x=c(\mathbf{A})$. Assume that $\delta(z)=1$, where $z \in \max (A ; \geq)$. By STEP 3, $c(\mathbf{A}) \in A_{m} \backslash A_{m-1}$. By LIIA, $x P y$ for any $y \in A_{m} \backslash A_{m-1} \backslash\{x\}$. Hence, $x$ is $P$-maximal in $A_{m} \backslash A_{m-1}$, which is the desired result.

Part (ii) is proved in a similar way. $\square$

The weak order $\geq$, the strict linear order $P$, and the priority indicator $\delta$ are unique since $(\geq, P, \delta) \neq\left(\geq^{\prime}, P^{\prime}, \delta^{\prime}\right)$ implies that $D_{\geq, P, \delta} \neq D_{\geq^{\prime}, P^{\prime}, \delta^{\prime}}$. Therefore, we conclude that $c$ is a $D_{\geq, P, \delta}$ 